
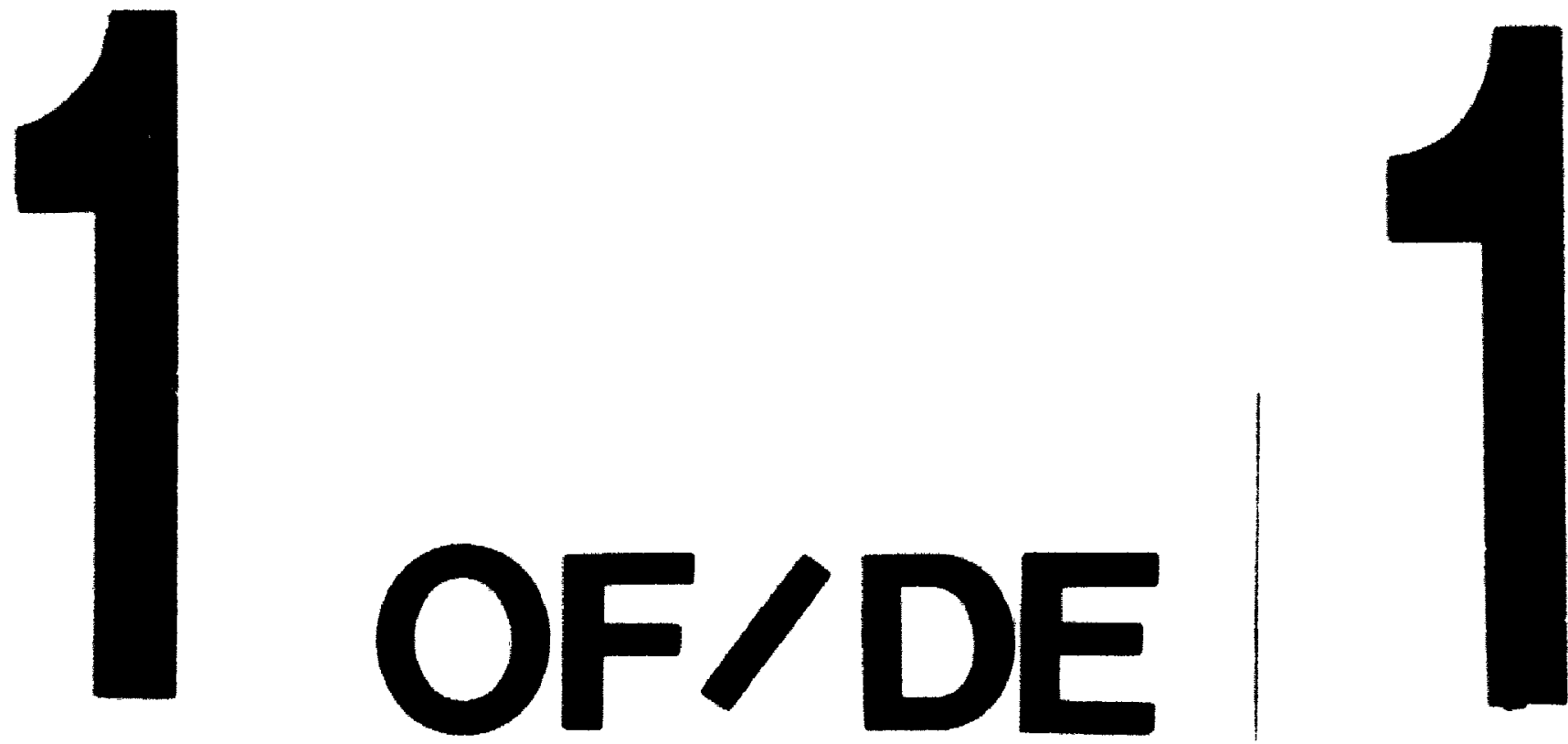

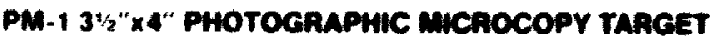
Nes rova AMst/150 "2 EOUIVALENT

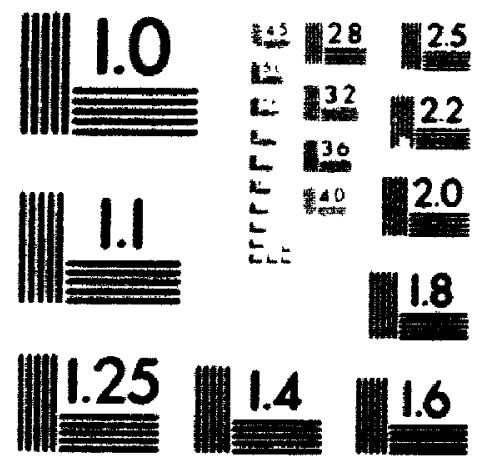


Acquisitions and

Bibliographic Services Branch

395 Weilngton Street

Otlawa. Ontario

KIA ONA
Biblothèque natıonale

du Canada

Direction des acquisitions at

des services bibliographiques

395. rue Weltington

Onawa (Ontaro)

\section{NOTICE}

AVIS

La qualité de cette microforme dépend grandement de la qualité de la thèse soumise au microfilmage. Nous avons tout fait pour assurer une qualité supérieure de reproduction.

S'il manque des pages, veuillez communiquer avec l'université qui a conféré le grade.

Some pages may have indistinct print especially if the original pages were typed with a poor typewriter ribbon or if the university sent us an inferior photocopy.

If pages are missing, contact the university which granted the degree.

$$
\text { qui a contéré le grade. }
$$

La qualité d'impression de certaines pages peut laisser à désirer, surtout si les pages originales ont été dactylographiées à l'aide d'un ruban usé ou si l'université nous a fait parvenir une photocopie de qualité inférieure.

La reproduction, même partielle, de cette microforme est soumise à la Loi canadienne sur le droit ci auteur, SRC 1970, c. C-30, et ses amendements subséquents.
Reproduction in full or in part of this microform is governed by the Canadian Copyright Act, R.S.C. 1970, c. C-30, and subsequent amendments. 


\title{
A FAST FOURIER TRANSFORM RADIX-2 COMPLEX BUTTERFLY WITH BUILT-IN SELF-TEST
}

by

lison W.K. Wong, B. Eng

\author{
A thesis submitted to \\ the Faculty of Graduate Studies and Research \\ in partial fulfilment of \\ the requirements for the degree of \\ Master of Engineering
}

Ottawa-Carleton Institute for Electrical Engineering

Department of Electronics

Carleton University

Ottawa, Ontario

December 1992

(C) copyright

1992, Wilson W.K. Wong 
National Library

of Canada

Acquisitions and

Bibliographic Senvices Branch

395 Wellington Street

Otawa Ontario

K1A ONA
Bibliotheque nationale

du Canada

Direction des acquisitions et

des services bibliographiques

395. nue Wellington

Onawa (Ontano)

Kia ONA
The author has granted an irrevocable non-exclusive licence allowing the National Library of Canada to reproduce, loan, distribute or sell coples of his/her thesis by any means and In any form or format, making this thesis available to interested porsons.
L'auteur a accordé une licence irrévocable et non exclusive permettant à la Bibliothèque nationale du Canada do reproduire, prêter, distribuer ou vendre des copies de sa these de quelque manière et sous quelque forme que ce soit pour mettre des exemplaires de cette thèse à la disposition des personnes intéressées.

L'auteur conserve la propriété du droit d'auteur qui protége sa these. Ni la thèse ni des extraits substantiels de celle-ci ne doivent être imprimés ou autrement reproduits sans son autorisation.

ISBN $\quad 0-315-84139-7$ 
The undersigned hereby recommend to the Faculty of Graduate Studies and Research acceptance of the thesis,

\section{A FAST FOURIER TRANSFORM RADIX-2 COMPLEX BUTTERFLY WITH BUILT-IN SELF-TEST}

submitted by

Wilson W.K. Wong, B.A.Sc.,

in partial fulfilment of the requirements

for the degree of Master of Engineering.

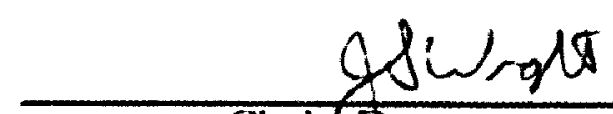

Chair Department of Electronics

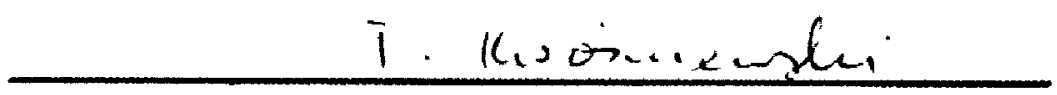

Thesis Supervisor

Carleton University

December 23, 1992 


\begin{abstract}
This thesis proposes a high-speed VLSI architecture for computing the radiv-2 complex butterfly. The architecture employs redundant binary arithmctics, parallel pipelining, and Booth's algorithm. A 16-bit x 12-bit, radix-2 complex butterfly has been implemented in a 15,000-gate, 180-pin pin-grid-array application-specific integrated circuit (ASIC). The ASIC technology is a 1.5-micron complementary-metal-oxide silicon (CMOS) gate-array. The twenty ASIC prototypes were a first-pass success, and test results show an average complex data sampling rate of $12.3 \mathrm{MHz}$ or $24.6 \mathrm{Msamples} / \mathrm{sec}$. Pairs of radix-2 complex butterf!v ASIC and the switch ASIC [1] can be grouped to form a high-speed, $49.2 \mathrm{Msamples} / \mathrm{sec}$ parallel pipeline fast Fourier Iransform (FFI) processor of sizes up to 32-point without the necessity of other integrated circuits (IC). or up to 8, 192-point with some external memory ICs. The target application of the high-speed FFT is in the multi-carrier group demodulator of an on-board satellite processor system. The complex butterfly ASIC also has built-in self-test (BIST) circuitry to assist chip-level and board-level testing.
\end{abstract}

The present complex butterfly architecture allows an easy migration path for increasing the data sampling rate by six folds to $75 \mathrm{MHz}$ or 150 Msamples/sec. 


\section{Acknowledgement}

I am grateful to: Dr. V. Szwarc for his initial idea of this project, Mr. L. Desormeaux for his assistance in prototype testing and characterization of the complex butterfly ASIC, and Communication Research Centre - Department of Communications Canada, Telecommunications Research Institute of Ontario, and NSERC for their funding. Most importantly, throughout the project, encouragement, support, understanding and patience received from Dr. C.H. Chan, Dr. T.A. Kwasniewski, my parents and my wife, Marysia are greatly appreciated. 


\section{Table of Contents}

1 INTRODUCTION............................................................................................ I

1.1 Thesis Motivation .................................................

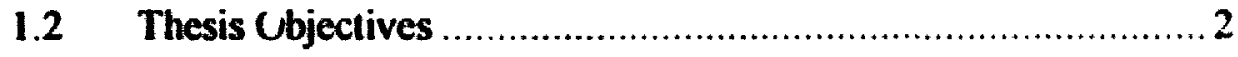

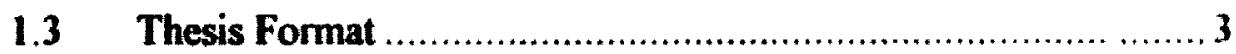

2 RADIX-2 COMPLEX BUTTERTLY AlgORITHM .............................. 4

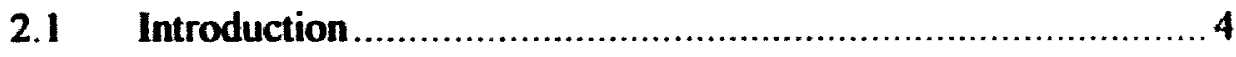

2.2 Discrete Fourier Transform ........................................ 4

2.3 Fast Fourier Transform Algorithm .............................. 5

2.4 DFT Computation Speed-up by FFT Algorithm.................6

2.5 Decimation-in-time Radix-2 Complex Butterfly Algorithm ....... 8

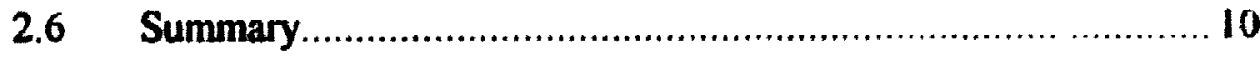

3 REDUNDANT BINARY ARITHMETICS ............................................. 11

3.1 Introduction ..........................................................

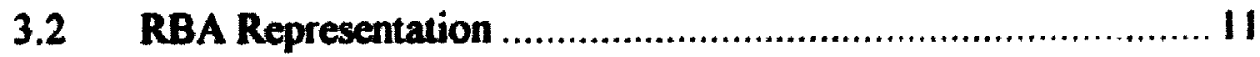

3.3 Format Conversion between RBA and 2's Complement ......... 12

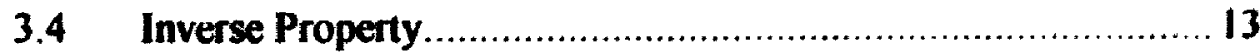

3.5 Carry Propagation-Free RBA Adder ......................... 14

3.6 RBA Adder Cell Logical Design ................................ 16

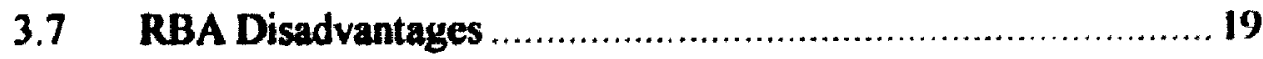

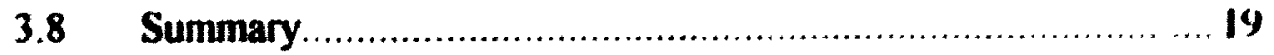




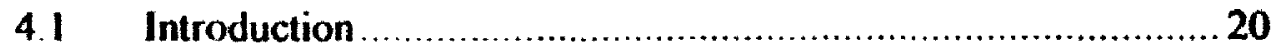

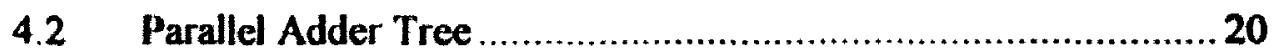

4.3 Booth's Multiplication Algorithm ................................ 21

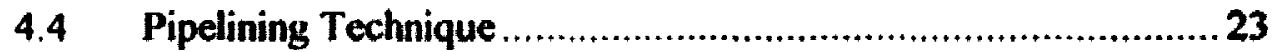

4.5 Radix-2 Complex Butterfly Architecture ..............................23

4.6 Complex Twiddle Factor Storage and Access Port ...................26

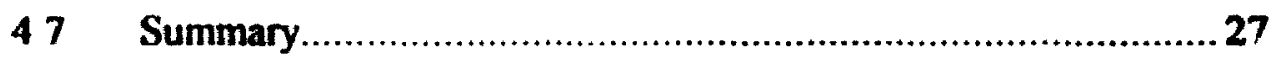

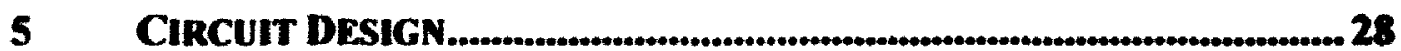

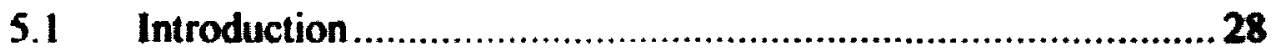

5.1 Booth's Decoder and Partial Product Generator .................... 28

$5.2 \quad$ Redundant Binary Adder ............................................. 30

5.3 Redundant to Binary Conversion...........................................30

5.4 Pipelining Speed Estimation ........................................ 32

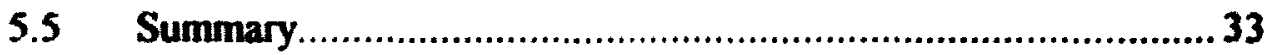

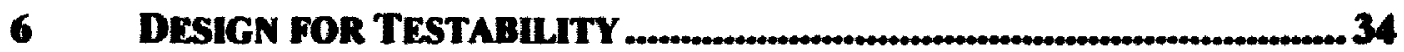

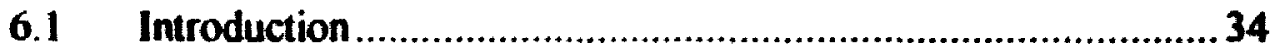

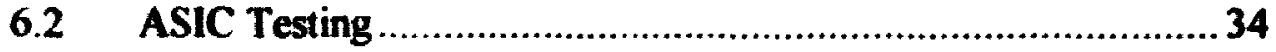

6.3 Design for Testability - Testing Strategies .......................... 35

6.4 Radix-2 Complex Butterfly ASIC Testing Strategy ................ 38

6.5 Pseudorandom Test Vector Generator................................. 39

6.6 Multiple Input Signature Analyzer................................40 40

6.7 BIST Gate Count .................................................... 41

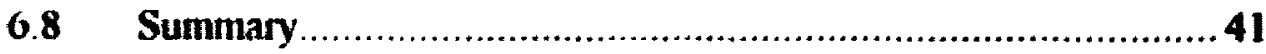


7 CMOS GATE-ARRAY TECHNOLOGY .............................................42

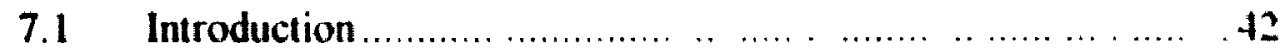

$7.2 \quad$ ASIC Computer-Aided Design Tools ........................ 42

7.3 Gate-array ASIC Back-End Design Process ........ . ...........4

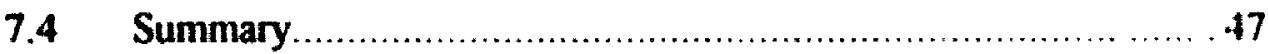

8 Fault Simulation Results and Prototype Test

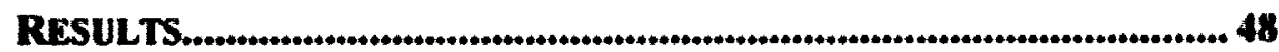

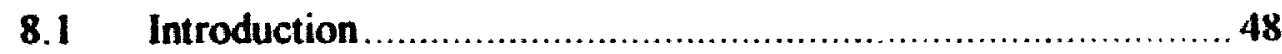

8.2 Fault Simulation Results ..................................................

8.2.1 Fault Simulation Result Discussion ......................... 48

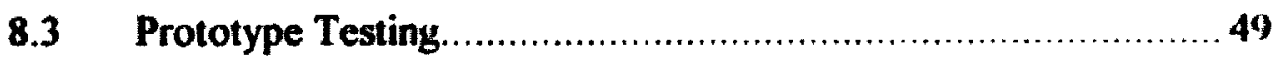

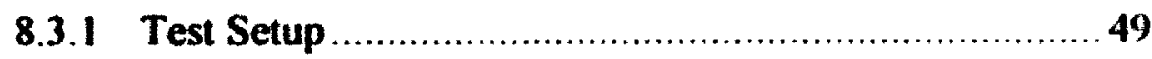

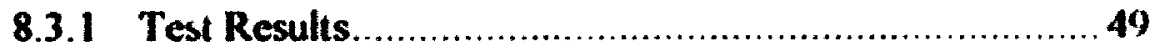

8.3.1 Test Result Discussion............................................50

8.4 Speed Comparison with Existing Reported Solutions ……........ 55

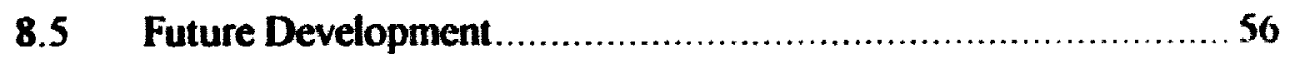

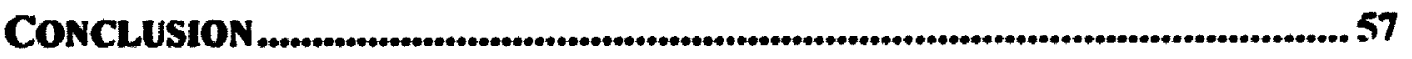

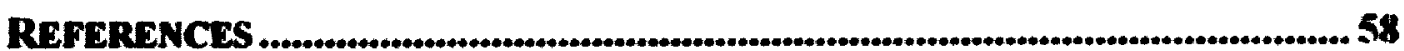




\section{List of Figures}

Figure 2-1: Data Flow Graph for 8-point Decimation-in-time FFT 7

Figure 2-2: Radix-2 DIT Complex Butterfly.................................................

Figure 3-1: Format Conversion from 2's Complement to Redundant Binary............. 12

Figure 3-2: Format Conversion from Redundant Binary to 2's Complement............ 13

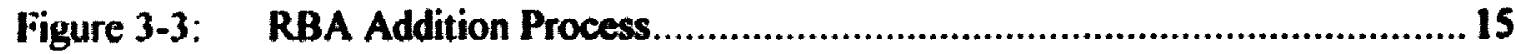

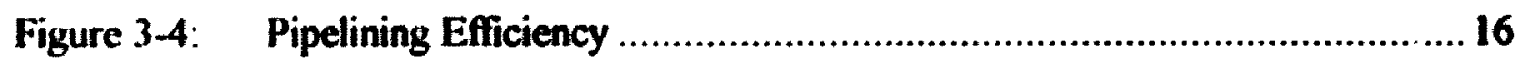

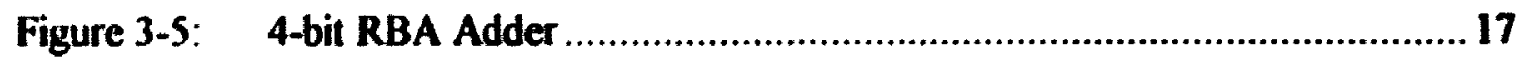

Figure 3-6: $\quad$ RBA Universal Adder Logical Design...................................... 18

Figure 4-1: (a) Shift and Add Multiply (b) Multiplier Summing Tree.....................21

Figure 4-2: Multiplication Example Using Booth's Algorithm.........................23

Figure 4-3: Complex Butterfly Pipeline Timing Diagram ...............................25

Figure 4-4: Complex Butterfly Architecture Block Diagram .............................25

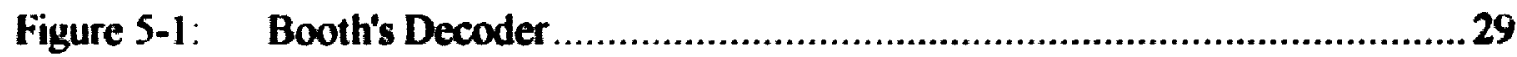

Figure 5-2: $\quad$ Partial Product Generator Bit-slice............................................. 29

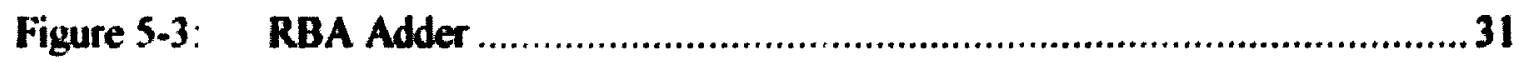

Figure 5-4: Redundant Binary to 2's Complement Conversion Bit-slice ................ 32 


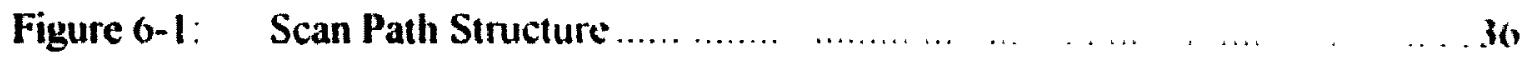

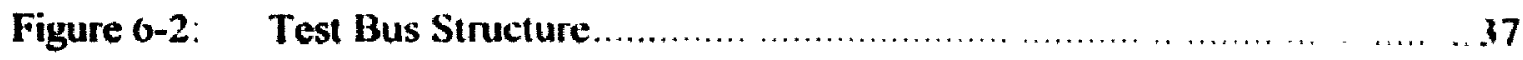

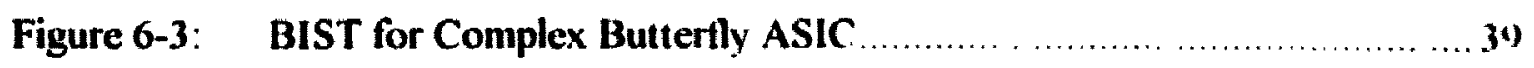

Figure 6-4: 16-bit LFSR and 12-bit LFSk Circuit Implementation ................. 40

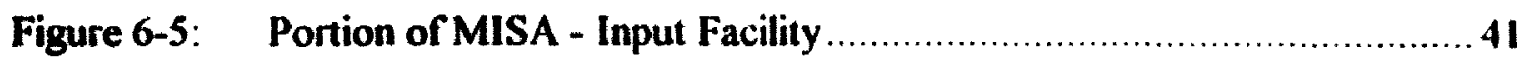

Figure 7-1: Bottom-Up Hierarchical Schematic Example. ...........................43

Figure 7-2: Example of Logic Functional Simulation ................................. 45

Figure 7-3: $\quad$ Data Setup and Hold-Time for Data Register.......................... 40

Figure 8-1: The Radix-2 Complex Butterfly ASIC ......................................53

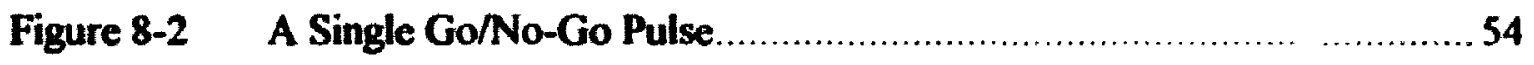

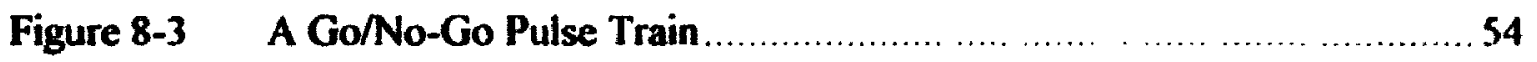




\section{List of Tables}

Table 2-1: DFT Computation Comparison Between Direct Method and FFT Algorithm . 8

Table 3-1: RBA Redundant Bits and Three-value Representation......................... 12

Table 3-2: Transfer Value and Intermediate Sum Value Selection Table................ 15

Table 4-1: Modified Booth's Algorithm Recoding Table .....................................22

Table 5-1: Redundant Binary to 2's Complement Conversion Table ........................ 32

Table 8-1: Measured and Adjusted Maximum Clock Frequency for 5V Case ........51

Talle 8-2: Measured Maximum Clock Frequency for 3.3V Case..........................52

Table 8-3: BIST Test Results for 3.3V and 5V ................................................55 


\section{Glossary of Abbreviations}

\begin{tabular}{ll} 
ASIC & Application-Specific Integrated Circuit \\
BIST & Built-In Self-Test \\
CMOS & Complententary-Metal-Oxide Silicon \\
DFT & Discrete Fourier Transform \\
DIT & Decimation-in-time \\
FFT & Fas' Fourier Transform \\
IC & Integrated Circuit \\
LFSR & Linear Feedback Shif Register \\
MISA & Multiple Input Signature Analyzer \\
NAND & Not-And Function \\
NSEC & Nano Second \\
PCB & Printed Circuit Board \\
RBA & Redundant Binary Arithmetic \\
ROM & Read Only Memory \\
\hline
\end{tabular}




\section{Chapter 1}

\section{Introduction}

\section{I Thesis Motivation}

FFT is an efficient algorithm in comparison to the direct method for evaluating discrete Fourier transform (DFT) [2,3]. The importance of the DFT is its transform property between time domain and frequency domain for sampled signal data. DFT applications have found their way into signal spectrum analyzer, sonar application, and radar signal processing [4,5]. Recently, with the advent of microelectronic technology and digital signal processing techniques, more contemporary applications have buen made possible. One of these applications is an on-board satellite multi-carrier group demodulator in which frequency division multiplexed signals from the up-link are demultiplexed - in a manner analogous to a white light being split into a colour (frequency) spectrum by a prism. Tire demultiplexed signals are then processed (sorted and recombined) into a time division multiplexed format and retransmitted down to the earth station $[6,7,8]$. This on-board satelite processing reverts the traditional satellite taak, which acts as a mirror for relaying signals over a long distance, to an intelligent satellite. With on-board processing, up-link transmission equipment can be made simpler, more compact, and affordable even for mobile users. One of the digital signal processing techniques tor the multi-carrier group demodulator is a polyphase filter combined with a FFT. To meet the data processing rate of $50 \mathrm{Msamples} / \mathrm{sec}$ - data rate required for an onboard satellite processing - FFT is implemented in an ASIC-based pipeline FFT structure. Faster than all other solutions $[9,10,11,12]$ - including the digital signal processor based FFT - ASIC-based pipeline FFT has been developed [13] to have a $10 \mathrm{MHz}$ data sampling rate. A recent research paper reports a wafer scale FFT processor that 
projects a $80 \mathrm{MHz}$ d.ta sampling rate [14]. The first motivation of this thesis is to develop a high-speed FFT radix-2 complex buttertly VLSI architecture. This architecture, when implemented with a 1.5 -micron 15,000-gate ASIC technology. is to target a data sampling rate of $12.5 \mathrm{MHz}$.

The radix-2 complex butterfly ASIC implemented in this thesis and the companion switch ASIC implemented in another thesis [1] aim to provire a minimum ASIC chip-set solution for the pipeline FFT. By cascading a number of these chip-set pairs, up to 32-point FFTs can be arranged to require no other IC, or up to 8,192-point that require some external memory ICs. To date, all the VLSI implementations reported for the radix-2 complex butterfly processor have involved a multiple-chip solution by Fox [13] and Plessey [15]. Among the reasons for a multiple-chip solution are economics - to kcep ASICs within a reasonable die size - and markcting - partitioning the complex butterfly into separate re-marketable ICs (complex-multiplier and complex-accumulator ASICs) for sale to other DSP applications. For the on-board satellite multicarrier group demodulator application where space, power, and speed are major considerations [16], a single-chip complex butterfly solution is more appropriate: the second motivation of this thesis.

\subsection{Thesis Objectives}

The first thesis objective is to develop a high-speed complex butterfly architecture. This thesis proposes the use of redundant binary arithmetic (RBA). The advantages of RBA ov $s$ the conventional binary arithmetic are its carry-free propagation property and sign-inverse property. These two properties are the main contributor to a high-speed complex butterfly architecture; the two properties result in a modular, systolic, and propagation-free adder design. 
The second thesis objective is to implement a 16-bit $\times 12$-bit, radix-2 complex butterfly chip based on the architecture developed. A 15,000-gate 1.5-micron rivios gatc-array is used for implementation. The target data sampling rate is $12.5 \mathrm{MHz}$.

The third thesis objective is to propose an efficient test strategy to perform chip-level and board-level tests with a high fault coverage and yet maintain minimal impact on the limited gate budget.

\subsection{Thesis Format}

In Chapter 2, the DFT and FFT algorithm are reviewed. The radix-2 complex butterfly algorithm and its characteristics relating to the architectural development are discussed. Chapter 3 provides the RBA basics: its advantages, disadvantages, and the logical implementation of an RBA adder. The RBA adder is also one of the main contributing factors in developing a high-speed architecture which is presented in Chapter 4. Before addressing the final architecture, three high-speed multiplication techniques employed in the architecture are highlighted. The high-speed architecture is a pipelining architecture; within the pipeline, are three types of circuit; the three types of circuit are described in Chapter 5. An inherent issue with ASIC design is its testability; ASIC teat issues and test strategies for this chip are discussed in Chapter 6. Chapter 7 provides an overview of the ASIC design process from the schematic to the prototype used for the complex butterfly ASIC. Chapter 8 furnishes and discusses both the fault simulation and prototype test results. This final chapter is followed by a conclusion. 


\section{Chapter 2}

\section{Radix-2 Complex Butterfly Algorithm}

\subsection{Introduction}

After reviewing the discrete Fourier transform, this chapter derives the fast Fourier transform algorithm based on the decimation-in-time approach [17]. The DFT computation speed-up advantage offered by the FFT algorithm is highlighted. Finally, the basic computing engine in the FFT algorithm - the focus of this thesis - the radix-2 complex butterfy is discussed. Its real equations and their significance in the use of the redundant binary representation and in hardware mapping into an efficient architecture for ASIC implementation are examined.

\subsection{Diserete Fourier Transform}

Discrete Fourier transform is a digital signal processing technique in which a sampled data sequence representing a signal in the time domain can be transformed to the frequency domain. The DFT operation resembles a prism that segregates the white light (time domain) into a colour spectrum (frequency domain). The inverse DFT transforms a signal from the frequency domain to the time domain and has similar operations as the forward DFT. Only the forward DFT is discussed here. The DFT of a sampled data sequence $\{x(n)\}, 0 \leq n \leq(N-1)$ is defined as:

$$
X(K)=\sum_{n=0}^{(N-1)} x(n) e^{-j(2 n / N) n K}, \quad K=\{0,1,2,3 \ldots .(N-1)\}
$$


where the $X(K)$ is the frequency domain sequence representing the signal and $X(n)$ is the time domain scxquence representing the identical signal.

\subsection{Fast Fourier Transform Algorithm}

FFT is an efficient way to calculate DFT [2]. FFT is a divide-and-conquer type of algorithm. It divides the original DFT intc two DFTs of equal length; each is then divided into two equal but shorter length DFTs. The successive dividing process repeats until the length of the divided DFT becomes a two-point DFT. This two-point DFT is called the radix-2 FFT.

To derive the FFT algorithm from the DFT algorithm, equation (1), the DFT formula can be rewritten as:

$$
X(K)=\sum_{n=0}^{(N-1)} x(n) W_{N}^{n K}, \quad W_{N}=e^{-j(2 \Pi / N)}
$$

In equation (2), $x(n) W_{N}^{n K}$ can be divided into its even and odd points as:

$$
X(K)=\sum_{\substack{n=0 \\ \text { even only }}}^{(N-1)} x(n) W^{n K}+\sum_{\substack{n=0 \\ \text { odd only }}}^{(N-1)} x(n) w^{n K}
$$

Alternatively it can be rewritten as: 


$$
X(K)=\sum_{n=0}^{(N / 2-1)} \times(2 n) W_{N}^{2 n K}+\sum_{n=0}^{(N / 2-1)} \times(2 n+1) W_{N}^{(2 n+1) K}
$$

because

$$
w_{N}^{2}=\left[e^{-j(2 \Pi / N)}\right]^{2}=\left[e^{-j(2 \Gamma / N / 2))}\right]=w_{N / 2}
$$

Substituting equation (5) into equation (4), it becomes,

$$
X(K)=\sum_{n=0}^{(N / 2-1)} x(2 n) W_{N / 2}^{n K}+W_{N}^{K} \sum_{n=0}^{(N / 2-1)} x(2 n+1) W_{N / 2}^{n K}
$$

Equation (6) shows that a length-N DFT can be replaced by two DFTs of length-(N/2) with the second DFT multiplied by a complex twiddle factor, $\mathbf{W}_{\mathbf{N}}^{K}$. This odd- and even-sequence way of dividing is called the decimation-in-time method. Figure 2-1 shows the data flow graph of an eight-point decimation-in-time, radix-2 FFT. The basic computation engine of radix-2 complex FFT is the radix-2 complex butterfly.

\subsection{DFT Computation Speed-up by FFT Algorithm}

In comparison with the number of complex multiplications, the direct computation of DFT shown in equation (1) requires $(\mathrm{N}-1)^{* * 2}$ complex multiplications versus the FFT computation of DFT requires $\mathrm{N} / 2^{*}\left(\log _{2} \mathrm{~N}\right)$ complex multiplications. Table 2-1 shows the FFT computation speed-up relative to the direct computation. 


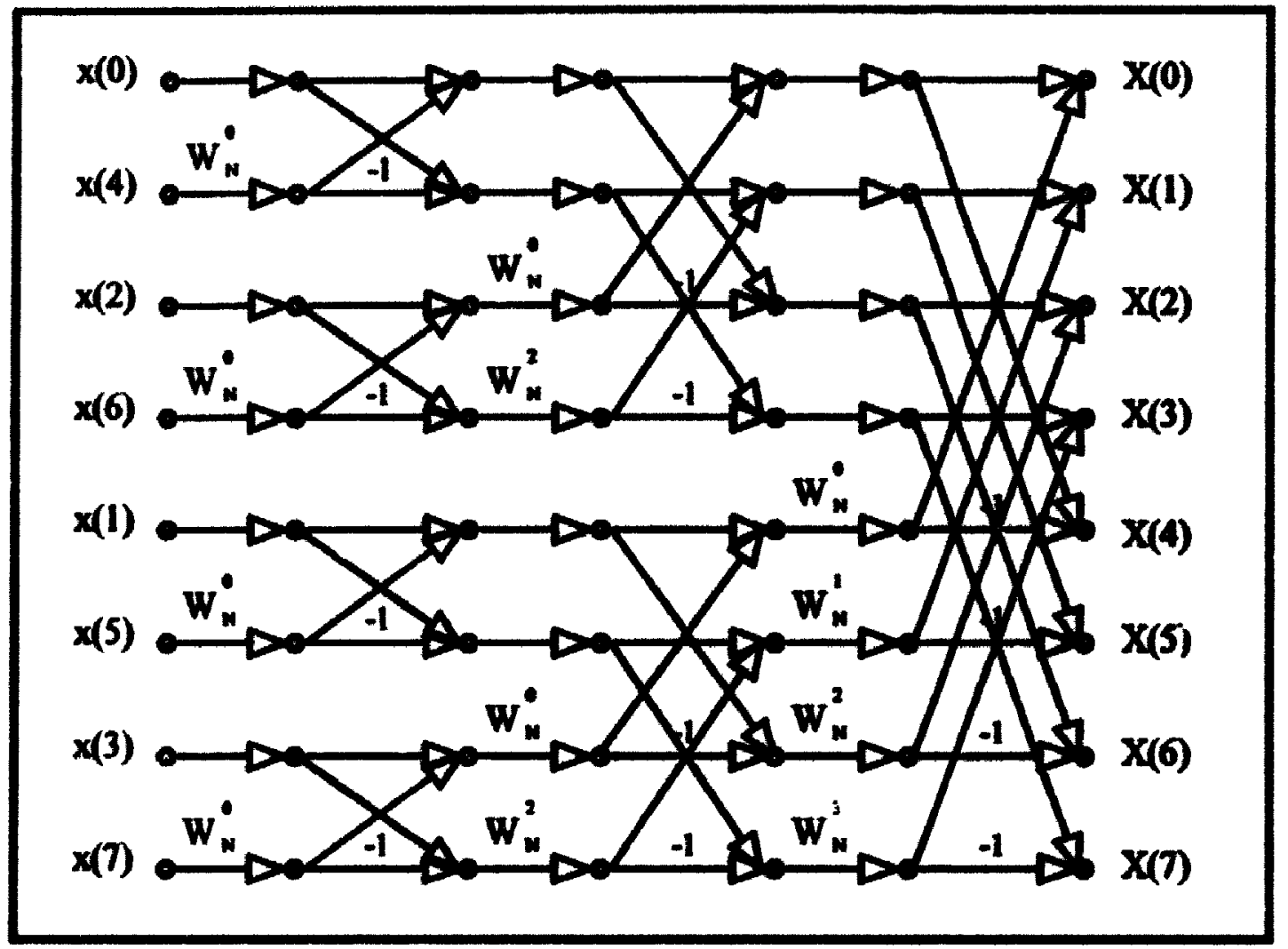

Figure 2-1: Data Flow Graph for 8-point Decimation-in-time EFT 


\begin{tabular}{|c|c|c|c|}
\hline $\begin{array}{c}\text { Number of } \\
\text { Points } \\
\text { N }\end{array}$ & $\begin{array}{c}\text { Complex Multiplications in } \\
\text { Direct Computation } \\
\text { N2 }\end{array}$ & $\begin{array}{c}\text { Complex Multiplications in } \\
\text { FFT Algorithm } \\
\text { N/2*(log } 2)\end{array}$ & $\begin{array}{c}\text { Speed- } \\
\text { up }\end{array}$ \\
\hline 4 & 16 & 4 & 4 \\
\hline 8 & 64 & 12 & 5 \\
\hline 16 & 256 & 32 & 8 \\
\hline 32 & 1024 & 80 & 12 \\
\hline 64 & 4096 & 192 & 21 \\
\hline 128 & 16384 & 448 & 36 \\
\hline 256 & 65536 & 1024 & 64 \\
\hline 512 & 262144 & 2304 & 113 \\
\hline 1024 & 1048576 & 5120 & 204 \\
\hline
\end{tabular}

Table 2-1: DFT Computation Comparison Between Direct Method and FIT

\section{Algorithm}

\subsection{Decimation-in-time Radix-2 Complex Butterly Algorithm}

The computation engine of radix-2 FFT is the radix-2 complex butterly. Figure 2-2 shows the equations for the DIT radix-2 complex buttenly in which the complex inputs and outputs are:

$$
\begin{aligned}
& x(0)=a+b j \\
& x(1)=c+d j \\
& x^{\prime}(0)=a^{\prime}+b^{\prime} j=x(0)+w_{N}^{K} * x(1)
\end{aligned}
$$




$$
x^{\prime}(1)=c^{\prime}+d^{\prime} j=x(0)-W_{N}^{K} * x(1)
$$

$$
x(1)=c+d j
$$

Figure 2-2: Radix-2 DIT Complex Butterfly

And

$$
W_{N}^{K}=e^{-j(2 n / N) K}=e^{-j \theta}=\cos \theta-j \sin \theta
$$

In real operations

$$
\begin{array}{ll}
a^{\prime}=a+c * \cos \theta-d * \sin \theta \quad \text { (real component) } \\
c^{\prime}=a-c * \cos \theta+d * \sin \theta \quad \text { (real component) } \\
b^{\prime}=b+c * \sin \theta+d * \cos \theta \quad \text { (imaginary component) } \\
d^{\prime}=b-c * \sin \theta-d * \cos \theta \quad \text { (imaginary component) }
\end{array}
$$

Two characteristics of these equations are relevant to using the redundant binary arithmetic and to mapping efficient hardware architecture for the ASIC implementation.

The first characteristic of these equations is the identical product terms between the real equations $a^{\prime}$ and $c^{\prime}$ and the imaginary equations $b^{\prime}$ and $d^{\prime}$, with only a difference in signs. This characteristic can take advantage of RBA's propagation delay-free sign-inverse operation. Once the product term is generated and using the RBA representation, both the 
positive and the negative products will be available simultaneously for parallel cvaluations of the real or imaginary equations.

The second characteristic of these equations is the overall similarity in all fiur equations - each equation has one single term and two product terms This characterstic allows a pipelining evaluation of the real equations which are then tollowed by the imaginary equations. This parallel and pipelining architecture will be discussed in Chapter 4.

\subsection{Summary}

This chapter has presented a derivation of the FFT algorithm for shortcut DFT computation; it has shown the computation speed-up achieved by the FFT algorithm.

Also discussed was the computing engine for the FFT algorithm - the focal point of this thesis - the radix-2 complex butterfly. The two characteristics in the four real equations have been pointed out for their important relevance to RBA and to hardware ASIC architecture mapping. 


\section{Chapter 3}

\section{Redundant Binary Arithmetics}

\subsection{Introduction}

This thesis proposes the use of RBA for designing a high-speed multiplieraccumulator radix-2 complex butterfly. That an RBA multiplier is faster than a standard array multiplier has been shown by Takagi [18]. RBA's modular and systolic adder cell allows for compact and efficient VLSI layout $[19,20]$.

This chapter covers three levels of redundant binary representation. The first level is the conceptual level of redundant binary representation and its relationship with the 23 complement binary representation. The second level is the RBA adder cell algorithm. The third level is the logical implementation of an RBA adder cell. At each level of redundant binary representation, the significance and importance related to the radix-2 complex butterfly algorithm and to the hardware architecture for the ASIC are highlighted. RBA's disadvantages are also discussed.

\subsection{RBA Representation}

A redundant binary system is an adapted binary system for implementing three-value logic $[21]$ in which each value can represent a value of $(+1,0,-1)$. The adapted binary system makes use of two binary bits (one positive redundant bit and one negative redundant bit) to make the representation. Table 3-1 shows the value represented by these two redundant binary bits. 


\begin{tabular}{|c|c|c|}
\hline $\begin{array}{c}\text { Positive } \\
\text { Redundant Bit }\end{array}$ & $\begin{array}{c}\text { Negative } \\
\text { Redundant Bit }\end{array}$ & $\begin{array}{c}\text { Value } \\
\text { Represented }\end{array}$ \\
\hline 0 & 0 & 0 \\
\hline 0 & 1 & -1 \\
\hline 1 & 0 & 1 \\
\hline 1 & 1 & invalid state \\
\hline
\end{tabular}

Table 3-1: RBA Redundant Bits and Three-value Representation

\subsection{Format Conversion between RBA and 2's Complement}

Conversion from 2's complement to RBA is straightforward. The negative redundant bit of the most significant bit position connects to the 2's complement sign bit. The remaining 2's complemem bits connect to their respective positive redundart bits. All other unconnected redundant bits are grounded. This conversion incurs no propagation delay. Figure 3-1 shows a conversion example from 2's complement format to redundant binary format.

Sign bit

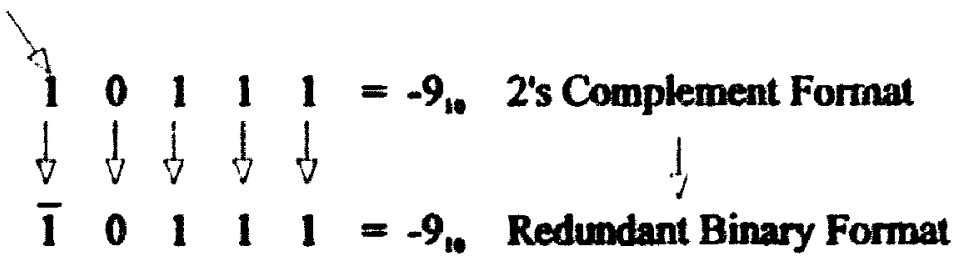

Figure 3-1: Format Conversion from 2's Complement to Redundant Binary 
Conversion from redundant binary format to 2 's complement format involves a separation and a subtraction operation. First, the negative redundant bits and the positive redundant bits are separated to form a negative number and a positive number. The negative number is subtracted from the positive number and the result is the converted 2's complement number. Figure 3-2 shows a conversion example from redundant bits to 2's complement format.

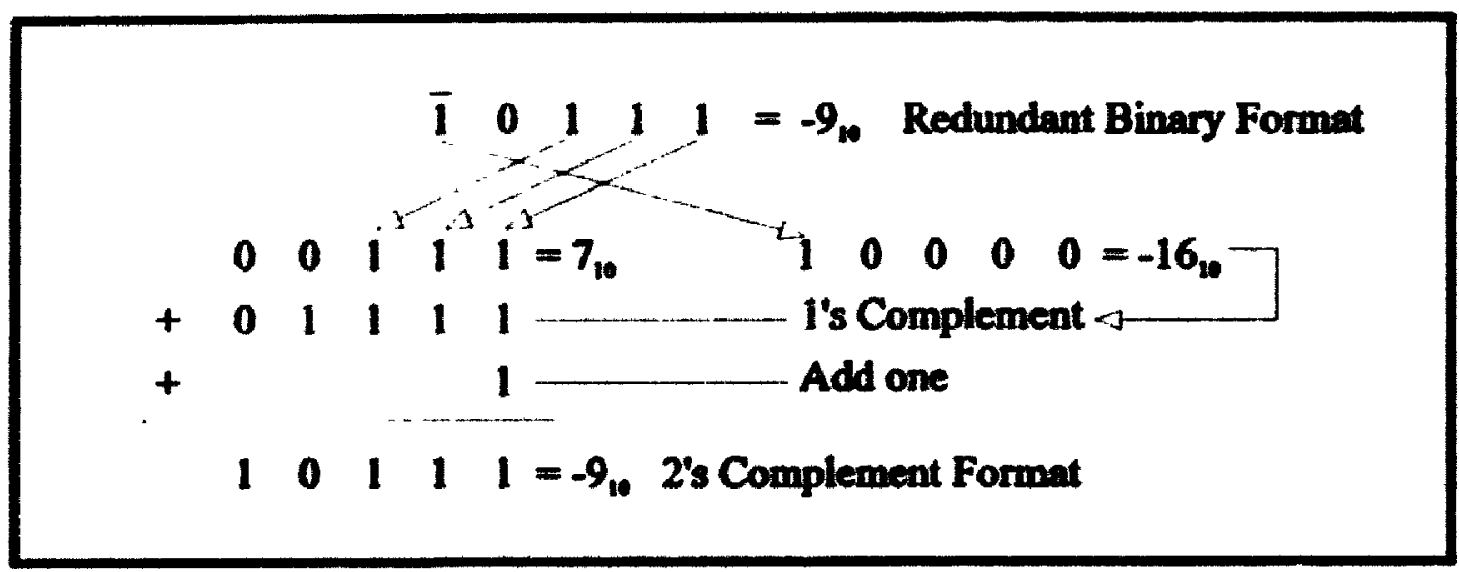

Figure 3-2: Format Conversion from Redundant Binary to 2's Comolement

\subsection{Inverse Property}

RBA has a convenient inverse property stemming directly from the redundant bits representation. In Table 3-1, the redundant bits representations for the values -1 and +1 are mirror-imaged. When the two redundant bits are swapped, the value is inverted.

In hardware, swapping the redundant bits can be performed by a simple signal wire exchange. Unlike the cumbersome procedure of forming 1's complement and then adding one in the 2's complement binary arithmetic, forming the inverse of a number is instantaneous and requires no propagation delay. 
This inverse property is a great asset for the complex buttertly ASIC in Iwo areas. One area is the high-speed Booth's multiplication algoritum, in which formation of negative partial products $(*-1, *-2)$ is required. The other area is in the IDIT complex butterfly algorithm, in which parallel evaluation of the real equations ( $a^{\prime}$ and $e^{\prime}$ ) and the imaginary equations ( $b^{\prime}$ and $d^{\prime}$ ) are made possible

\subsection{Carry Propagation-Free RBA Adder}

How does an RBA adder operate? An RBA adder takes two steps to ensure carry propagation-free additions. The first step is a selection process. For each bit position. a transfer-bit and an intermediate sum-bit are determined. In the second step, the intermediate sum bit and the transfer bit from the next lower bit position are added to form the sum for that bit position. Figure 3-3 illustrates this two-step RBA addition process.

Table 3-2 shows the rules for the selection process. Based on the values of the augend and addend $(x, y)$ for the present bit position and on the sum of augend and addend from the next lower bit position, the transfer value $\left(T_{(i)}\right)$ and the intermediate sum value $\left.W_{(i)}\right)$ are determined. The selection process guarantees that if there is a possible carry $\left(T_{i-1}=1\right)$ from the next lower position, it will be squashed by the intermediate sum value $\left(W_{i}=-1\right)$. Similarly, a possible borrow $\left(T_{i-1}=-1\right)$ will be squashed by the intermediate sum value $\left(W_{i}=1\right)$. Figure 3-3 gives a working example of an 8-bit $\mathrm{KBA}$ adder. 


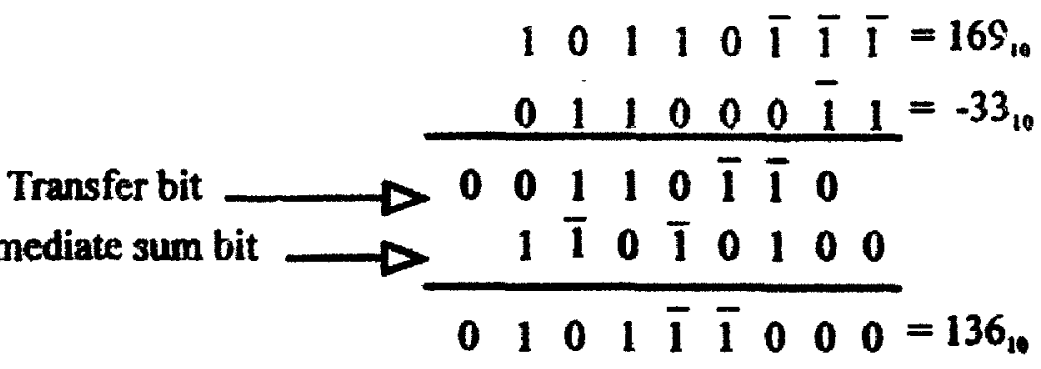

Figure 3-3: RBA Addition Process

\begin{tabular}{|c|c|c|c|c|}
\hline $\begin{array}{l}\text { Augend } \\
\text { Value } \\
x(i)\end{array}$ & $\begin{array}{l}\text { Addend } \\
\text { Value } \\
\text { y(i) }\end{array}$ & $\begin{array}{l}\text { Sum of Augend and Addend } \\
\text { Values at the next lower position } \\
\qquad x(i-1)+y(i-1)\end{array}$ & $\begin{array}{l}\text { Transfer } \\
\text { Value } \\
\text { T(i) }^{\text {Tol }}\end{array}$ & $\begin{array}{l}\text { Int. Sum } \\
\text { Value } \\
\text { W(i) }^{\text {Vum }}\end{array}$ \\
\hline $\begin{array}{l}1 \\
0 \\
\end{array}$ & $\begin{array}{l}0 \\
1 \\
\end{array}$ & if sum $<0$ (no possible carry) & 0 & 1 \\
\hline $\begin{array}{l}1 \\
0 \\
\end{array}$ & $\begin{array}{l}0 \\
1 \\
\end{array}$ & if sum $\geq 0$ (possible carry) & 1 & -1 \\
\hline $\begin{array}{c}-1 \\
0 \\
\end{array}$ & $\begin{array}{r}0 \\
-1 \\
\end{array}$ & if sum $\geq 0$ (no possible borrow) & 0 & -1 \\
\hline $\begin{array}{c}-1 \\
0 \\
\end{array}$ & $\begin{array}{c}0 \\
-1 \\
\end{array}$ & if sum $<0$ (possible borrow) & -1 & 1 \\
\hline 1 & 1 & Don't care & 1 & 0 \\
\hline-1 & -1 & Don't care & -1 & 0 \\
\hline $\begin{array}{r}1 \\
-1 \\
\end{array}$ & $\begin{array}{c}-1 \\
1\end{array}$ & Don't care & 0 & 0 \\
\hline
\end{tabular}

Table 3-2: $\quad$ Transfer Value and Intermediate Sum Value Selection Table 
Because of the carry propagation-free RBA adder. addition can be perfiomed in a constant time delay, independent of word-length This constant time delay is an important element for making a high-speed pipeline architecture, especially in which each pipeline stage requires a different word-length $\mathrm{RBA}$ adder. If a conventional carry propagation binary adder is applied, the pipeline speed will be dictated by the longest word-length adder due to the carry propagation. Figure $3-4$ shows the diflerence in pipelining efticiency (pipeline idle time) between equal delay tasks and unequal delay tasks

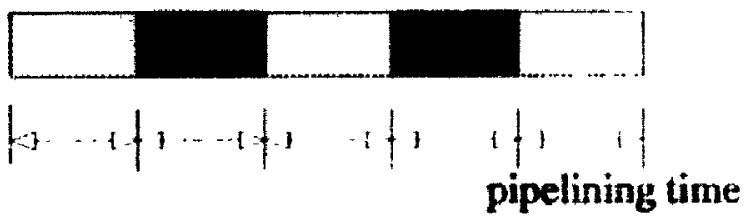

Tasks with equal time

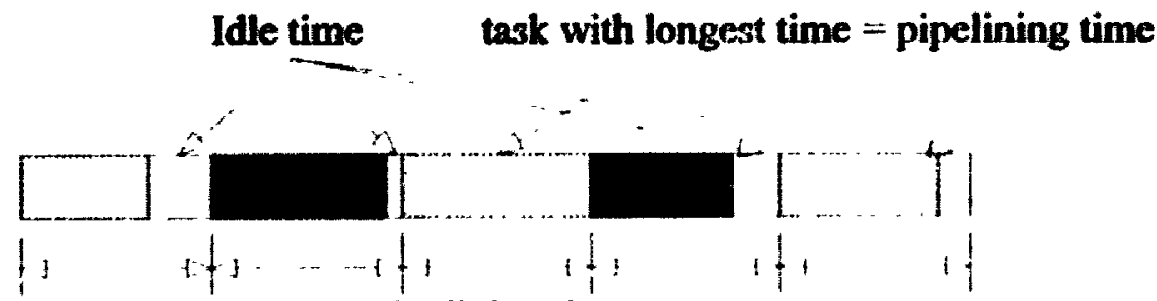

pipelining time

Tasks with unequal time

Figure 3-4: Pipelining Efficiency

\subsection{RBA Adder Cell Logical Design}

The RBA adder cell is universal for addition and subtraction. It accepts two redundant binary inputs (positive or negative number), and, with additional input from the next lower bit position, it produces two redundant binary sum bits Only next neighbour cells are involved for all data signals to and from the RIBA adder cell which can be 
classified as a systolic cell. The systolic cell has important implications in VLSI or ASIC implementation:

- by a tiling operation, it allows building of an adder of any word-length

- tiling makes RBA adders physically compact on the silicon

- compactness, in turn, minimizes interconnect metal tracking and tracking propagation delay for the RBA adder on the silicon; and consequently, it yields a high-speed adder.

Figure 3-5 shows the data-flow block diagram of a 4-bit systolic RBA adder.

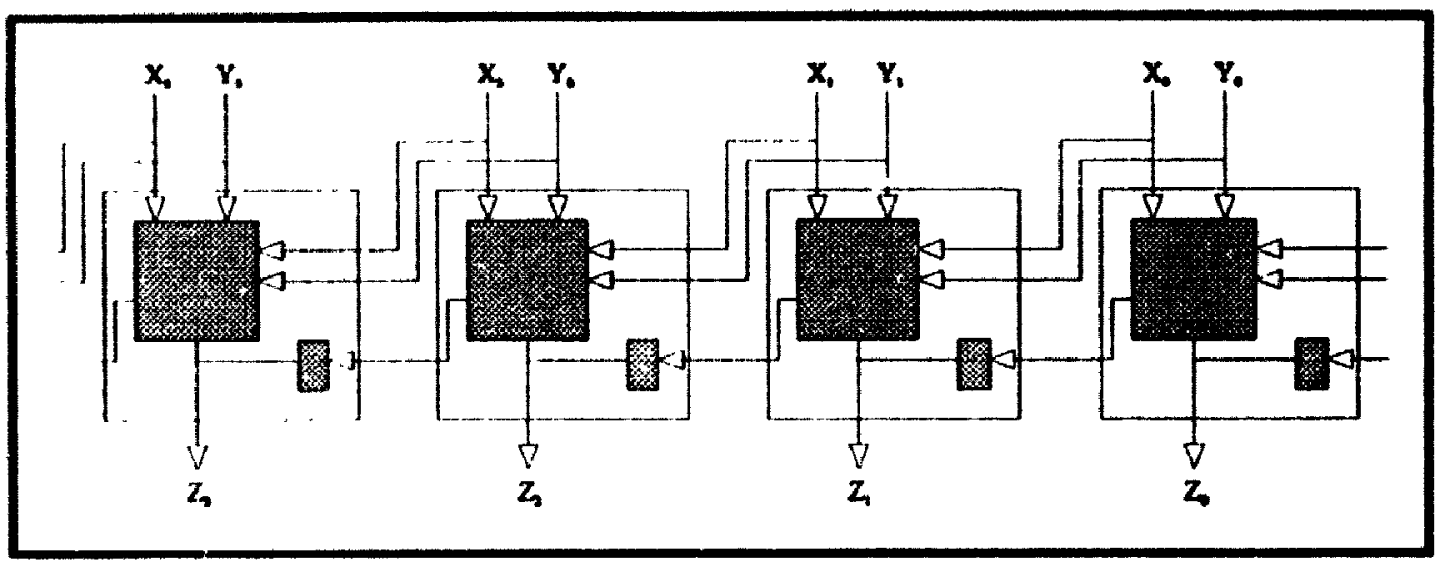

Figure 3-5: 4-bit RBA Adder

To move on to the logical implementation level of the RBA adder cell, Figure 3-6 shows the logical design of the RBA adder cell. The and-or gates are optimized macro-cell specific to the LSI 9K gate-array library; the gates have a propagation delay approximately similar to one gate level logic. The propagation delay for four-level logics is approximately 6 nsec in the 1.5-micron CMOS gate-array technology. 


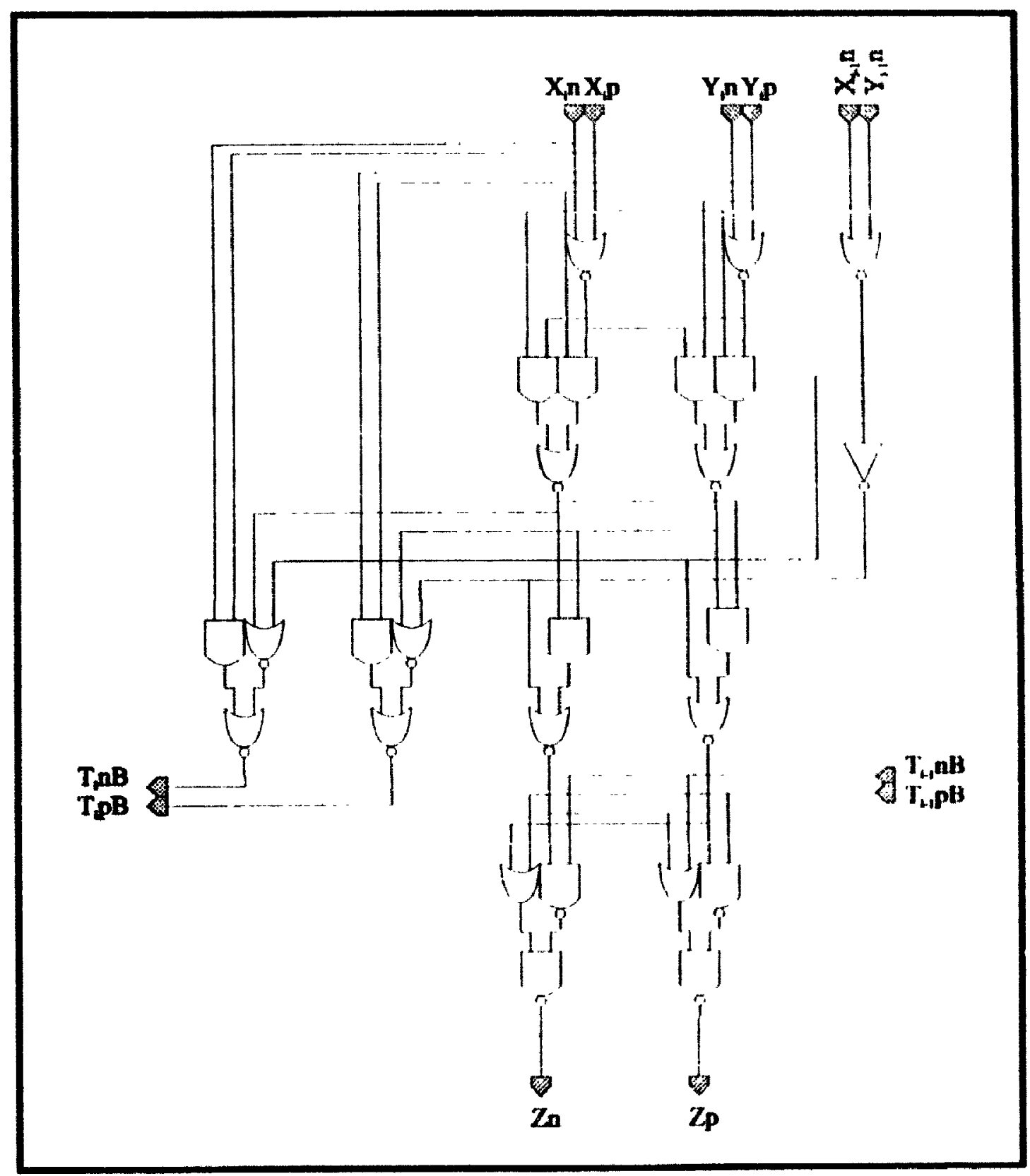

Figure 3-6: RBA Universal Adder Logical Design 


\subsection{RBA Disadvantages}

Although RBA has been shown to be effective in the previously mentioned arithmetic operations and hardware implementations, there are some disadvantages with RBA. One disadvantage is the doubling of gate count. The second disadvantage is the conversion to 2 's complement format at the data interface point.

The gate count doubling disadvantage is caused by the redundant logic in an RBA adder when implemented in a two-value logic CMOS technology. This disadvantage represents an implementation technology misfit rather than an inherent problem with the three-level logic. This disadvantage would disappear if the RBA adder could be implemented in some three-value logic technology.

If a three-level logic technology were available, format conversion could be greatly minimized. Three-level signals could be passed among complex butterfly ASICs. The format conversion from the three-level signal to a binary signal would only be required at the user interface point

\subsection{Summary}

This chapter has addressed the representation level, the algorithmic level, and the logical implementation level of the RBA. It has shown that the RBA inverse property is beneficial for Booth's multiplication algorithm and the radix-2 complex butterfly algorithm. It has also shown that the RBA's carry propagation-free property gives rise to systolic adder cell design and efficient pipelining architecture. In the next chapter, these constant delay adders will be used in the complex butterfly pipeline architecture. The RBA's disadvantages were also noted. 


\section{Chapter 4}

\section{Radix-2 Complex Butterfly ASIC Architecture}

\subsection{Introduction}

In this chapter, the radix-2 complex butterfly algorithm discussed in Chapter 2 is mapped to a parallel pipeline architecture. This chapter first discusses the three high-speed multiplication techniques employed in the pipeline architecture before detailing the parallel pipeline architecture. The ability to double the present data throughput rate by further parallelism - duplicating the present proposed architecture - is also suggested.

\subsection{Parallel Adder Tree}

The first high-speed multiplication technique is in the use of a parallel adder tree. Basic multiplication is generally performed by using a shif and add technique. Figure 4-1(a) shows that for every bit of the multiplier, a partial product is generated. The sum of these partial products equals the product.

In hardware multiplier implementation, a parallel summing tree can speed-up the multiplication by dividing the partial products and summing them in parallel. Figure 4-1(b) illustrates an 8-bit multiplier that uses a parallel summing tree. Compared to the basic serial summing approach, a parallel summing tree speeds up multiplication by a factor of $\sqrt{\mathrm{N}}$, where $\mathrm{N}$ is the word-length of the multiplier. 


\section{1 \\ $\times 011011$ \\ 010111 \\ 010111 \\ 000000 \\ 010111 \\ 010111 \\ 000000 \\ 01000010001}

(a)

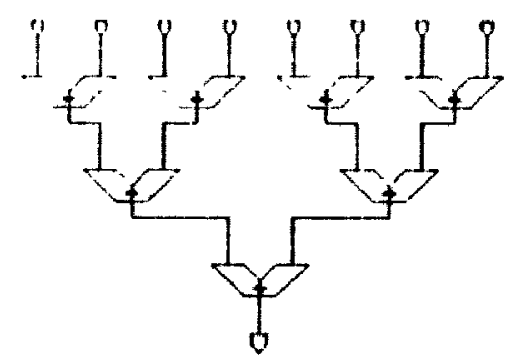

(b)

\section{Figure 4-1: (a) Shift and Add Multiply (b) Multiplier Summing Tree}

\subsection{Booth's Multiplication Algorithm}

The second high-speed multiplication technique is in the use of Booth's multiplication algorithm.

In binary representation, strings of ones in a number can be recoded as a minus one at the bottom of the string and a plus one at one position next to the top of the string. For example,

$$
00,1111,0001,1110_{2}=01,000 \overline{1}, 0010,00 \overline{10}_{2}
$$

if the above example is a binary multiplier. Before recoding, eight partial products are required (eight *1), but after recoding only four partial products are required (two *1 and two *-1). Recoding strings of ones can result in a lower number of partial products and in faster multiplication speed.

Such searching and skipping over variable lengths of strings of ones is difficult for hardware implementation. Booth's algorithm, is a more systematic search-and-skip recoding algorithm. 
Booth's algorithm examines the multiplier with a three-bit wide window that skips across two bits at a time. Table 4-1 shows the possible recoded outputs based on the contents in the 3-bit wide window. Instead of generating one partial product per bit of the multiplier, Booth's algorithm generates one partial product every two bits of the multiplier. The. efore, Booth's algorithm speeds up multiplication by a factor of two.

\begin{tabular}{|c|c|c|c|c|}
\hline \multicolumn{3}{|c|}{$\begin{array}{l}\text { Multiplier Bits } \\
\text { in the 3-bit } \\
\text { Window }\end{array}$} & \multirow[t]{2}{*}{$\begin{array}{l}\text { Recoded } \\
\text { Output }\end{array}$} & \multirow[t]{2}{*}{ Comments } \\
\hline$x_{i+1}$ & $x_{i}$ & $x_{i-1}$ & & \\
\hline $\mathbf{0}$ & 0 & 0 & $*(0)$ & zero strings \\
\hline 0 & 0 & 1 & $*(+1)$ & top of string of ones \\
\hline 0 & 1 & 0 & $*(+1)$ & single one / no string \\
\hline $\mathbf{0}$ & 1 & 1 & $*(+2)$ & top of string of ones \\
\hline 1 & 0 & 0 & $*(-2)$ & bottom of string of ones \\
\hline 1 & $\mathbf{0}$ & 1 & $*(-1)$ & $\begin{array}{l}\text { top and bottom of string of } \\
\text { ones }\end{array}$ \\
\hline 1 & 1 & $\underline{0}$ & $*(-1)$ & bottom of string of ones \\
\hline 1 & 1 & 1 & $*(0)$ & middle of string of ones \\
\hline
\end{tabular}

\section{Table 4-1: Modified Booth's Algorithm Recoding Table}

Figure 4-2 gives an example of multiplication that uses Booth's recoding algorithm. Using the RBA inverse property makes *-1, and *-2 partial product generation simple and direct: *-2 is don by a shifting left and then exchanging the redundant bit signals, while *-1 is done by exchanging redundant bit signals only. 


$$
\begin{aligned}
& 010111 \\
& 0110[1]=*-1 \longrightarrow \frac{\times 011011}{00 \overline{1} 0 \overline{1} \overline{1}} \\
& 01[0] 1=*-1 \longrightarrow 0 \text { İ⿴囗十 } \\
& \text { bDilo11 }=*+2 \frac{010111}{010100 \overline{1} 00 \overline{1}}
\end{aligned}
$$

\section{Figure 4-2: Multiplication Example Using Booth's Algorithm}

\subsection{Pipelining Technique}

So far two high-speed multiplication methods have been discussed. One other high-speed technique is called pipelining.

Pipelining is analogous to multi-stage assembly line in which the complete assembly task is partitioned into a series of sub-tasks. The output from one task is the input for the next task. The throughput speed of this assembly line is governed by the sub-task that requires the longest time. If all sub-tasks take equal time, there is no idle time in the assembly line. If however, a sub-task takes an unusually long time, thus dictating the pipelining time, there are idle periods in the assembly line for other shorter time sub-tasks.

In the radix-2 complex butterfly, all three high-speed multiplication techniques are employed: modified Booth's algorithm, parallel adder tree, and pipelining.

\subsection{Radix-2 Complex Butterfly Arehiteeture}

This section shows the hardware mapping of the radix-2 complex butterfly that uses the high-speed multiplication techniques described in the previous sections. 
A re-examination of the DIT radix-2 complex butterfly equations from Chapter 2 shows:

$$
\begin{array}{ll}
a^{\prime}=a+c * \cos \theta-d * \sin \theta \quad \text { (real component) } \\
c^{\prime}=a-c * \cos \theta+d * \sin \theta \quad \text { (real component) } \\
b^{\prime}=b+c * \sin \theta+d * \cos \theta \quad \text { (imaginary component) } \\
d^{\prime}=b-c * \sin \theta-d * \cos \theta \quad \text { (imaginary component) }
\end{array}
$$

These four equations include four product terms: $c^{*} \cos \theta, c^{*} \sin \theta, d * \cos \theta$, and $d^{*} \sin \theta$, and two single terms, a and b. Using RBA's inverse property, the real equations pair ( $a^{\prime}$ and $c^{\prime}$ ) and the imaginary equations pair ( $b^{\prime}$ and $d^{\prime}$ ) can be evaluated in parallet: except for the opposite sign, in the equations pair, each equation has the same single term and identical product terms. The parallel operation is indicated by the split operations inside a single time slot as shown in Figure 4-3: Complex Butterly Pipeline Timing Diagram. There is a choice to evaluate the real equations pair and the imayinary equations pair in parallel. However, due to constraint in gate resources, they are evaluated in a pipeline fashion. Figure 4-3 shows the pipeline operation and timing of the real equations pair and the imaginary equations pair.

Figure $4-4$ shows the parallel pipeline architecture for the radix-2 complex butterfly computation.

1) In the first pipeline stage, the 12-bit wide twiddle factor multiplier $(\cos \theta)$ is recoded by the booth decoder; six partial products for (c) are generated. 


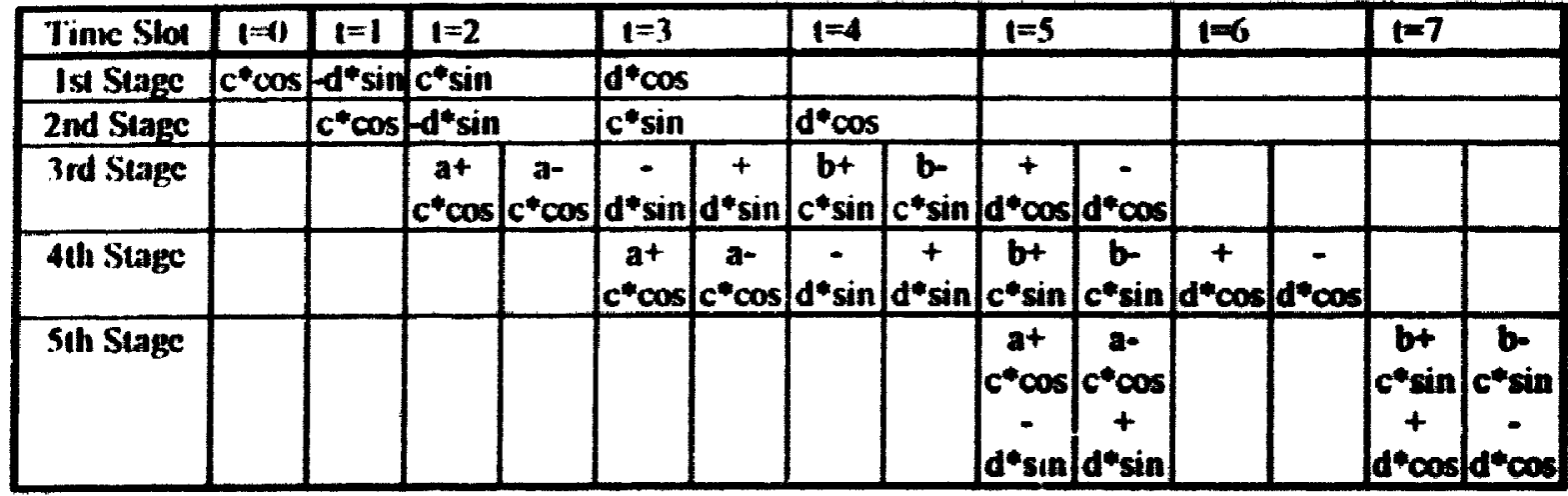

Figure 4-3: Complex Butterfly Pipeline Timing Diagram

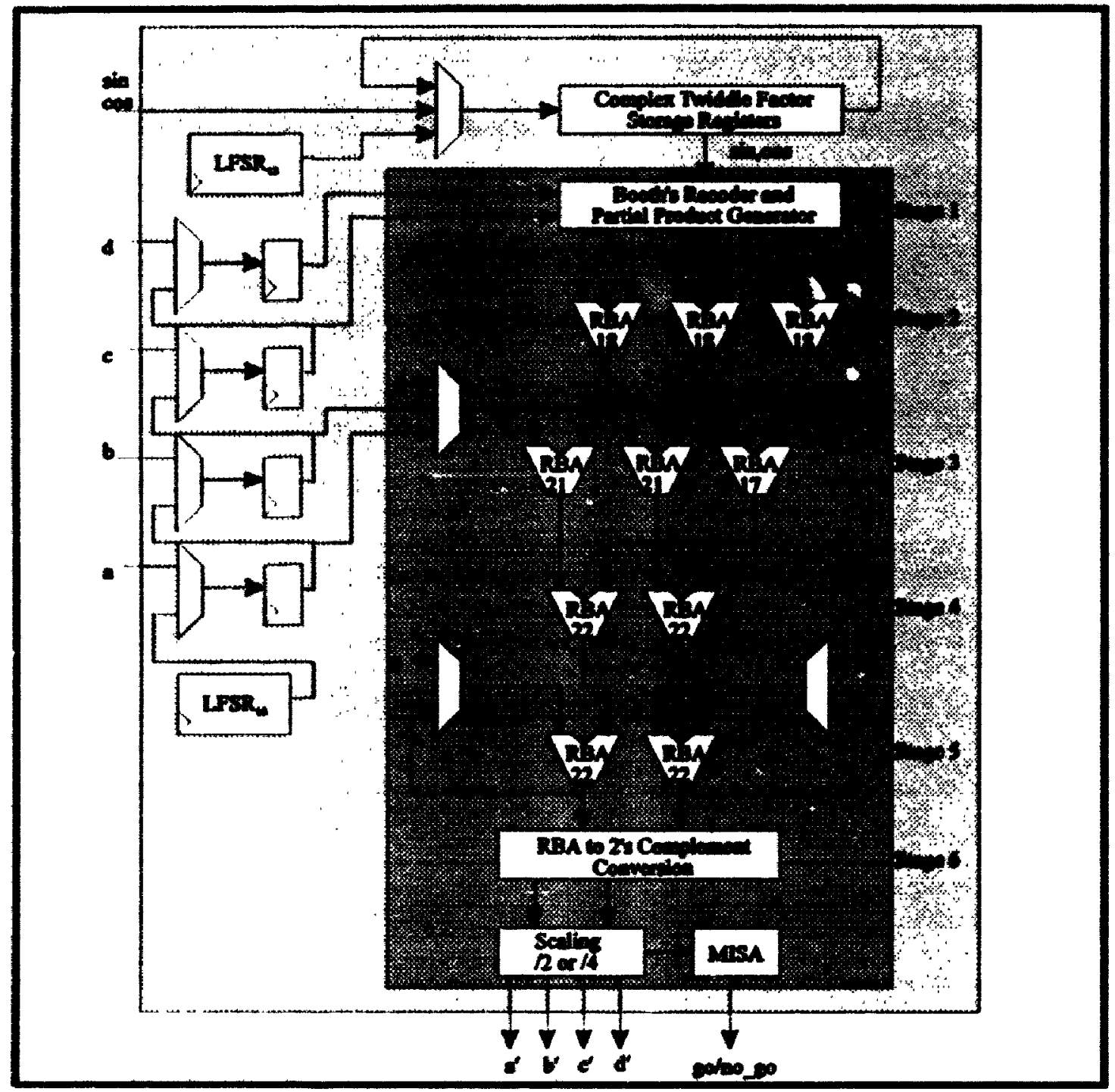

Figure 4-4: Complex Butterfly Architecture Block Diagram 
2) The second pipeline stage is an RBA adder stage at which partial products for $\left(+c^{*} \cos \theta\right)$ are summed. Partial products are reduced from six to three.

3) In the third stage, the single term (a) is introduced into two parallel pipelines: one pipeline computes $(a+c * c o s())$ and the other pipeline computes $\left(a-c^{*} \cos \theta\right)$. Notice that the $\left(-c^{*} \cos \theta\right)$ is generated by exchanging redundant binary bit signals. As well, there is a third RBA adder that sums up two remaining partial products of $\left(+c^{*} \cos \theta\right)$.

4) In stage four, the partial products of $(+c * \cos \theta)$ are added into the parallel pipelines. For the subtracting pipeline, the redundant binary bit signals are exchanged to generate the $\left(-c^{*} \cos \theta\right)$.

5) In stage five, $\left(a+c^{*} \cos \theta\right)$ and $\left(a-c^{*} \cos \theta\right)$ are fed back to the imput of the adder and are summed with the $\left(-d^{*} \sin \theta\right)$ and $\left(+d^{*} \sin \theta\right)$ which arrive one pipelining clock later.

6) The sixth pipeline stage converts $\left(a+c^{*} \cos \theta-d^{*} \sin \theta \quad a^{\prime}\right)$ and $\left(a-c^{*} \cos \theta+d^{*} \sin \theta=c^{*}\right)$ into 2 's complement fornat

7) The final stage is for optional scaling by two or by four and for cutput registering.

The computations for $b^{\prime}$ and $d^{\prime}$ are similar and they follow immediately be'iand $a^{\prime}$ and $c^{\prime}$ as depicted in the pipeline timing diagram, Figure 4-3

If $\left[a^{\prime}\right.$ and $\left.c^{\prime}\right]$ and $\left[b^{\prime}\right.$ and $\left.d^{\prime}\right]$ can be compuled in parallel by duplicating the parallel pipeline architecture discussed above, the data throughput rate will double

\subsection{Complex Twiddle Factor Storage and Access Port}

The $\sin \theta$ and $\cos \theta$ are the real and imaginary components of the complex iwiddle factors. They can be accessed from any of the eight internal registers (12-bit complex 
twiddle factor registers) or from an external parallel port. The on-chip twiddle factor storage is sufficient for a 32-point FFT application. However, above the 32-point FFT, external memory is accessed through the external twiddle factor access port.

\subsection{Summary}

A high-speed architecture for the radix-2 complex butterfly has been developed. Based on the inverse property and the propagation delay free property of the RBA, the radix-2 complex butterfly is mapped into a parallel and pipeline architecture. The advantage of the proposed architecture - ability to double the present data throughput rate by simple duplication of the proposed architecture - has also been suggested. 


\section{Chapter 5}

\section{Circuit Design}

\subsection{Introduction}

In Chapter 4, the radix-2 complex butterly algorithm has been mapped into a parallel pipeline architecture. Exclusive of the input and output register stages, three types of circuit are contained in the pipelining stages of this architecture. These three circuits are Booth's decoder and partial product generators, RBA adders, and redundant binary to 2's complement converters. This chapter provides the circuit design for all three pipeline elements. Estimation of the pipelining speed by the number of levels of logic in each circuit is given.

\subsection{Booth's Decoder and Partial Product Generator}

As discussed in Section 4.3, Booth's algorithm is used to halve the number of partial products. It decodes three multiplier bits and generates control signals for the partial product generation $(* 0, * 1, * 2,-1, *-2)$. Figure 5-1 shows the Booth's decoding circuit: b2, b1, and b0 are the multiplier bits; a flip input inverts the sign of the partial product which is used when performing $\left(-d^{*} \sin \theta\right)$; negative, two, and one are the output signals to the partial product generator.

Figure 5-2 shows a bit-slice partial product generator: $b(i)$ is the present bit and $b(i-1)$ is the next lower significant bit; one, two, and negative are the control signals from the Booth's decoder; bn and bp are the negative and positive redundant binary bit respectively for the present bit position. 


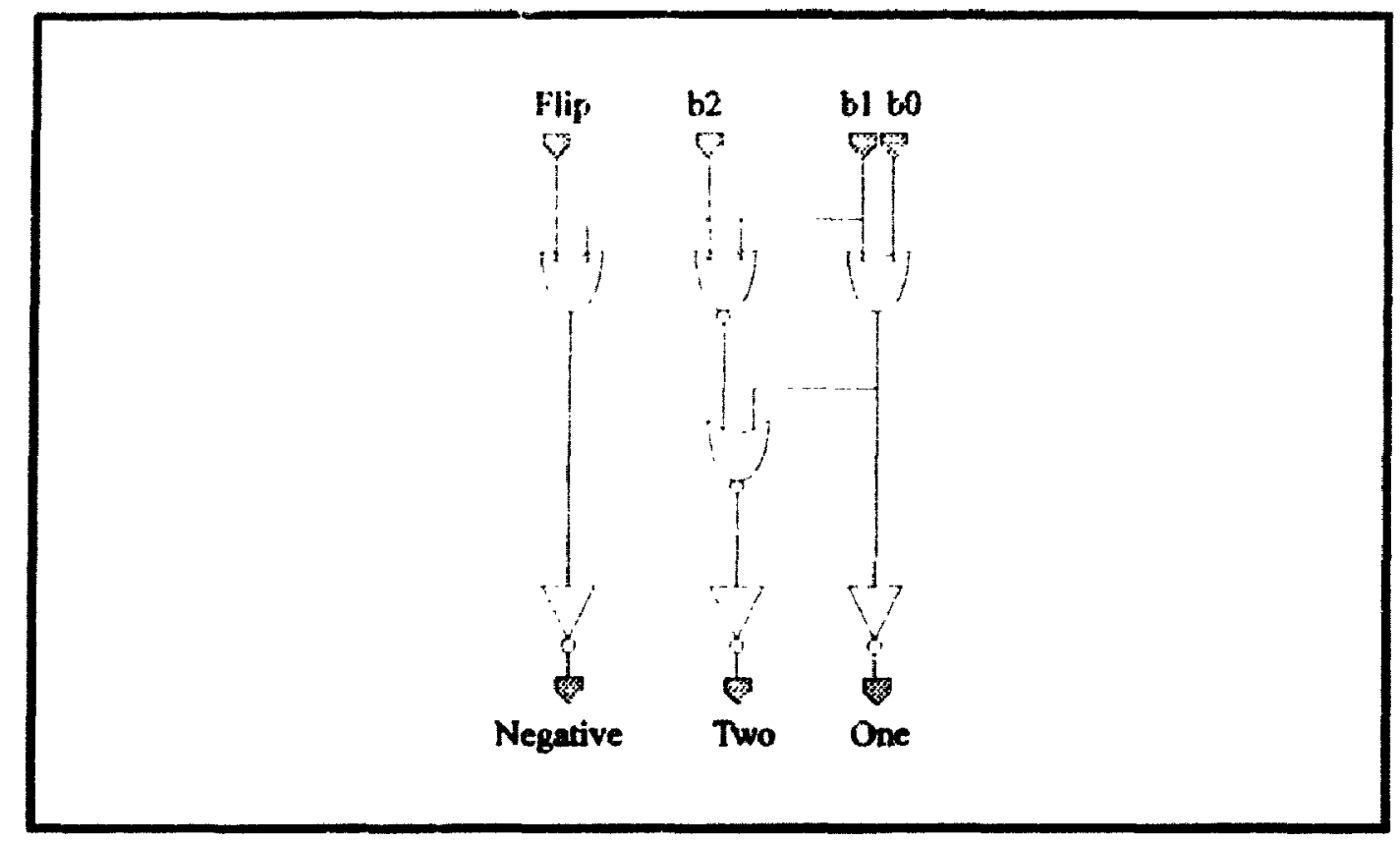

Figure 5-1: Booth's Decoder

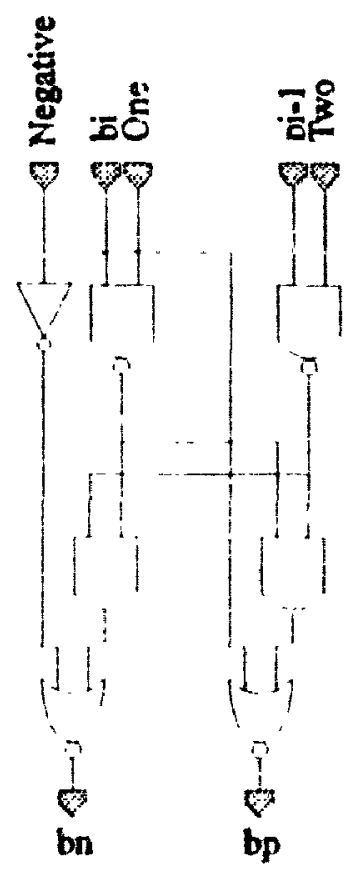

Figure 5.2: Partial Product Generator Bit-slice 
Booth's decoder and the partial product generator are in the first pipeline stage and have a total of five levels of logic.

\subsection{Redundant Binary Adder}

From pipeline stage two to pipeline stage five are the RBA adders. RBA adder las four levels of logic. Figure 5-3 shows the logical design of the RIBA adder; injuts include the two redundant addends, the negative red'ındant bits, and the transfer bits from the next lower significant position; outputs are the redundant sum and transfer bits.

\subsection{Redundant to Binary Conversion}

The conversion from the redundant binary format to the 2's complement format is performed in stage six of the pipeline architecture. The conversion can be performed over two pipeline clock periods because only one conversion is required for every two pipeline clock periods as shown in Figure 4-3, Complex Butterfly Pipeline Timing Diagram.

The conversion algorithm is a form of pattern recognition and is given in Table 5-1. When each redundant bit position is a non-zero, and if the first non-zero bit in the lower position is a negative one, then this bit is converted to a zero; otherwise, if the first non-zero bit in the lower position is a positive one, it is converted to a one When the bit is a zero and if the first non-zero bit in the lower position is a negative one, then this bit is converted to a one; otherwise, if the first non-zero bit in the lower position is a positive one, it is converted to a zero.

Figure 5-4 shows the conversion circuit for the fourth bit position

After conversion, if overflow or underflow is detected, the result is locked to the maximum or minimum value respectively. The number of logic levels of the redundant to binary converter is twelve. 


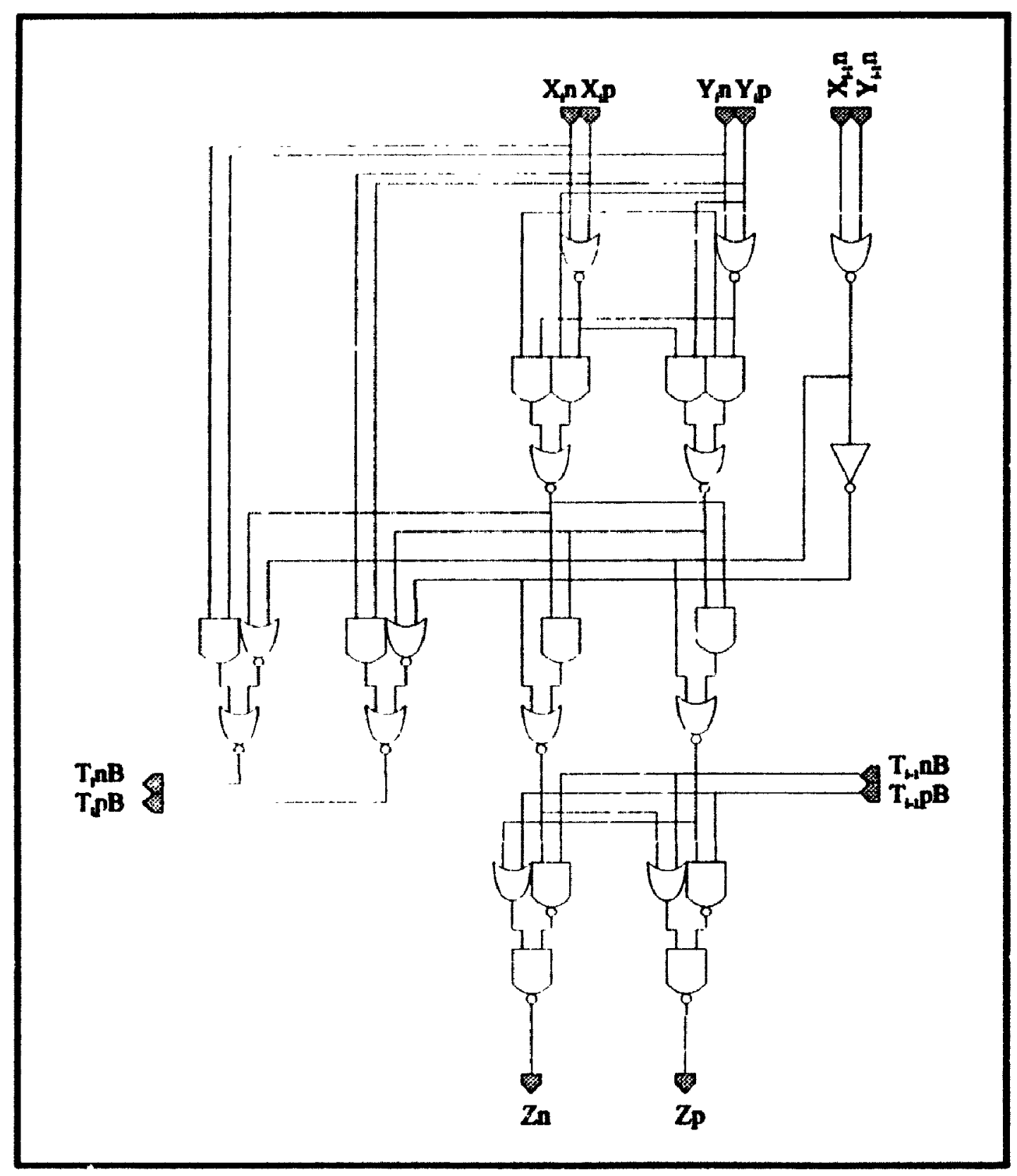

Figure 5-3: RBA Adder 


\begin{tabular}{|c|c|c|}
\hline Redundant Binary Bit & $\begin{array}{c}\text { When First Non-zero Bit in } \\
\text { the Lower Position }\end{array}$ & 2's Complement Bit \\
\hline non-zero $(1$ or -1$)$ & -1 & 0 \\
\hline non-zero $(1$ or -1$)$ & +1 & 1 \\
\hline 0 & -1 & 1 \\
\hline 0 & +1 & 0 \\
\hline
\end{tabular}

Table 5-1: Redundant Binary to 2's Complement Conversion Table

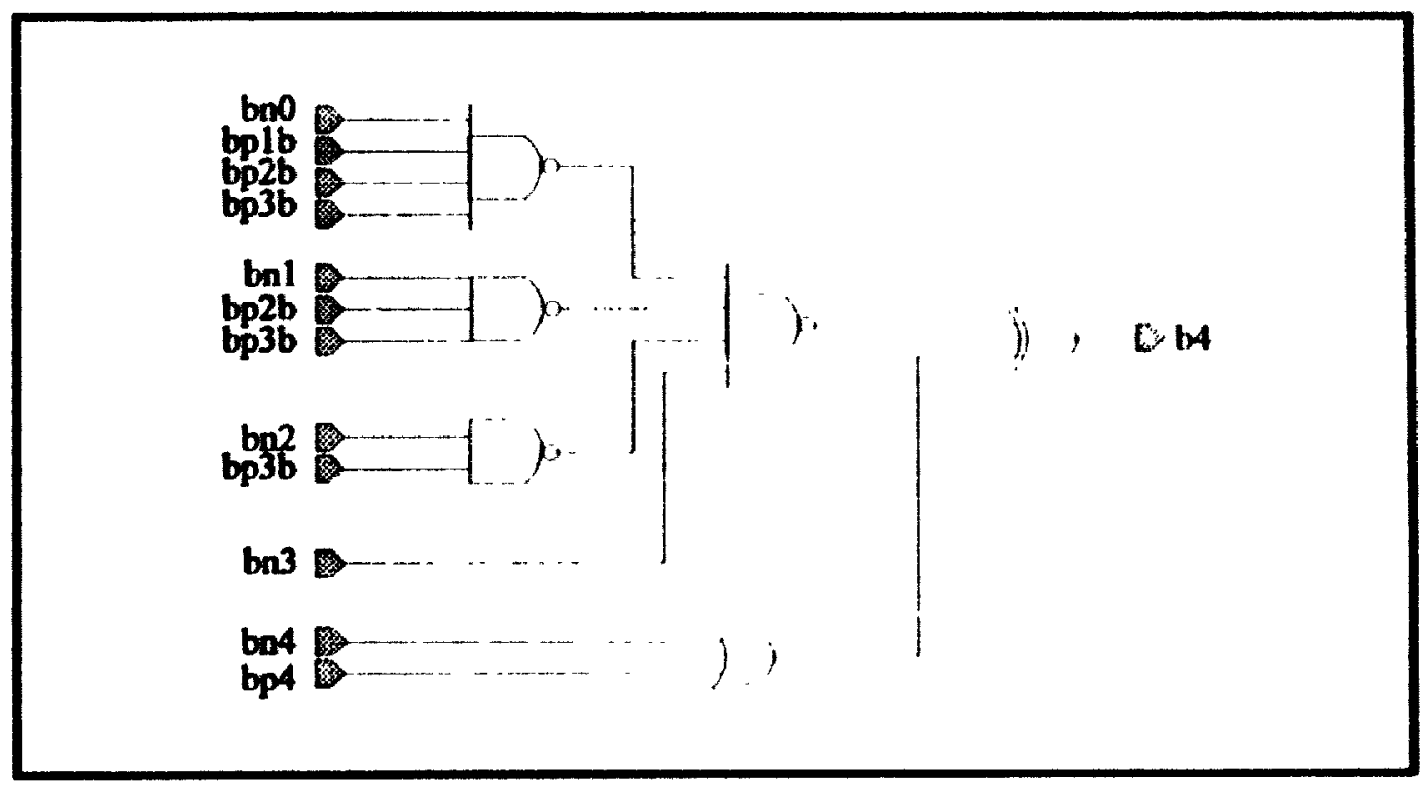

Figure 5-4: Redundant Binary to 2's Complement Conversion Bit-slice

\subsection{Pipelining Speed Estimation}

Five, four, and six are the number of logic levels for the Booth's decoder and partial product generator, the RBA adder, and the redundant to binary convertor. That they are all closely matched means that the pipelining is cficient and that there will be little idle time. Typical delay for each gate level is approximately $1.5 \mathrm{nsec}$. The input bulfer delay and the output buffer delay are approximately 4 and 3 nsec. The pipeline register 
delay (clock to output delay) is approximately $1.5 \mathrm{nsec}$. Therefore, the total delay including the input buffer delay, clock to output delay, logic level delay and output delay is $17.5 \mathrm{nsec}$. With a $20 \mathrm{nsec}$ target pipelining clock period $(50 \mathrm{MHz})$, there is a margin of 2.5 nsec

\subsection{Summary}

This chapter has shown the circuit design for Booth's decoder and partial product generator, RBA adder, and redundant to binary convertor; all are housed in the parallel pipeline architecture. Also shown is that the number of logic levels among Booth's decoder and partial product generator, RBA adder, and redundant to binary convertor are five, four, and five respectively from which a pipelining speed is estimated to be $50 \mathrm{MHz}$ which meets the speed target. 


\section{Chapter 6}

\section{Design for Testability}

\subsection{Introduction}

This chapter describes the approach to ASIC verification and the prevalent fault model for grading test vertors that are used for the verification process. As the size and the complexity of ASICs grow, testing problems emerge: the observability and controllability of internal nodes are increasingly difficult and the size of test vectors also rises. Some solutions to the ASIC testing problem which are collectively called "design for testability" are reviewed. At the end, the special test circuits (BIST) implemented in the complex butterfly ASIC are detailed.

\subsection{ASIC Testing}

ASICs have become a highly complex electronic device that contains from 10,000-gate and upward to 500,000-gate that target a specific functionality and that provides both a high-speed and compact circuits. These tiny silicon devices provide no visual inspection and no probing access; the only access to the ASIC is through the package pins. ASIC testing relies solely on exercising the ASIC through the pins by test vectors (input stimuli) and then on comparing the results to the expected results. Test vectors are the only means by which a designer can verify that the ASIC is defect free. Because ASIC vendors generally guarantee that the delivered chis: will pass the specified test vectors, it becomes essential that good fault coverage be attained by these test vectors. The prevalent method of determining fault coverage is through stuck-at fault simulation which hypothesizes two types of faults: either stuck-at-1 or stuck-at-0 for every signal net (gate level output). Many sources of manufacturing defects continue to range 
from device level defects - substrate surface defects, particle contamination, uneven substrate doping causing transistor threshold voltage error, and discontinuity of polysilicon gate - to the interconnect level defects - discontinuity of the metal track, metal to metal shorts, and signal line shorts to the supply rail or ground $[22,23]$. The stuck-at fault is deficient in modelling all the possible faults, but it is a means to quantifying the fault coverage and hence the effectiveness of test vectors.

Because of the high amortization cost for IC testers, ASIC vendors limit the size of the test vectors for manufacturing test (LSI Logic allows three sets of test vectors, each 4,000-vectors). This restriction presents a problem for complex ASIC testing.

Complex ASIC generally has a high gate-to-pin ratio which means that many internal nodes need to be tested with a relatively small number of package pins. The ability to control (to set up a fault detection through input pins) and to observe (to propagate the fault effect to output pins) faults becomes a monumental but not impossible task, though the size of test vectors will definitely not meet the vendor's restrictions. In the next section, solutions for this problem are described.

\subsection{Design for Testability - Testing Strategies}

The solution to the ASIC's high gate-to-pin ratio problem is the use of special test circuits promoted under "design for testability" methodology. By incorporating dedicated test circuits into an ASIC, so as to increase the internal visibility (controllability and observability) of the ASIC, compact test vectors writing becomes possible.

For example, for a finite state machine type ASIC, it would normally take a very long time to exhaust all the states; e.g., a simple 13-bit counter has 8,192 combinations. By putting in a full-scan chain, or partial-scan chains. state values can be scanned in and scanned out directly [24]. Figure 6-1 shows special scan flip flops (or data If ousters) 
chained together to form a scan path. Through this scan path, logic levels at these internal register nodes can be scanned in and scanned out via dedicated test pins and thereby improve internal controllability and observability to the ASIC. While scan design can achieve up to $100 \%$ stuck-at fault coverage, the price paid for full scan design can be as high as $20 \%$ of the silicon real estate.

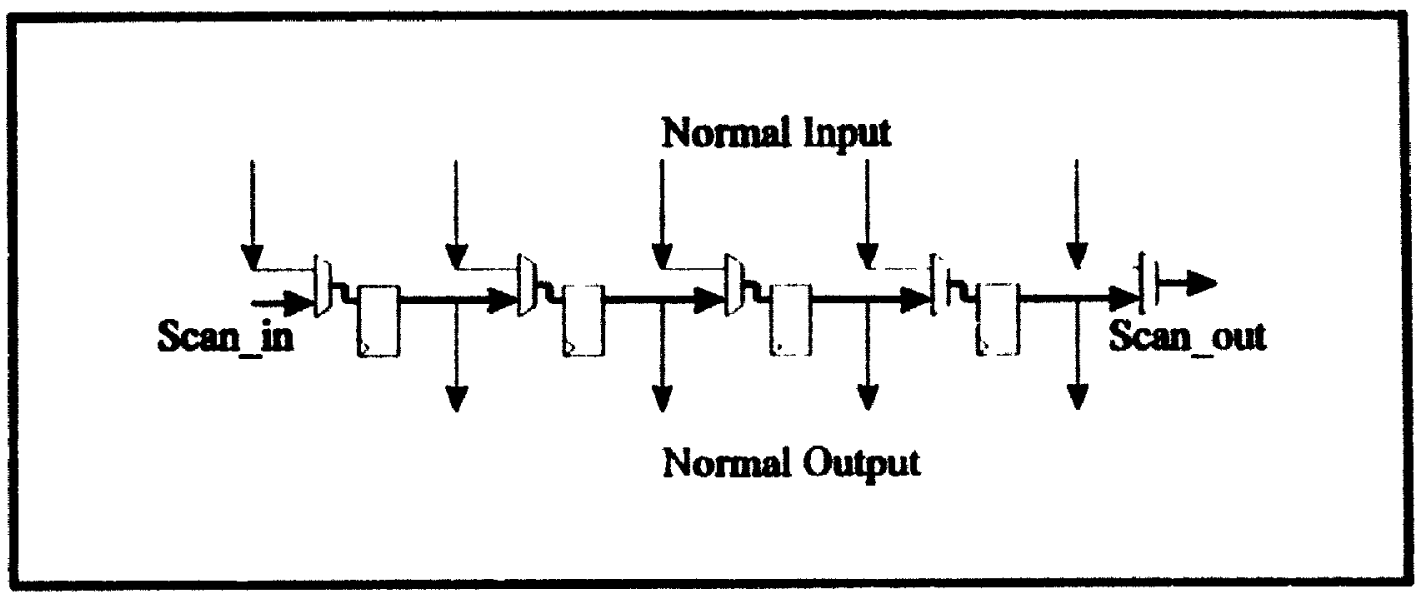

\section{Figure 6-1: Scan Path Structure}

Another type of test circuit is the test bus scheme which is suitable for a system on a chip type of ASIC. Figure 6-2 shows large functional modules that are threaded together by a parallel test bus. As a result, direct access to these internal blocks is possible, and the ASIC is partitioned into smaller block for testing, effectively reducing the gate-to-pin ratio.

An extension of the "design for testability" circuit is the built-in self-test. BIST is a self-contained on-chip testing package which includes test vector generation and signature analysis. Generally, there are two types of test patterns. Deterministic test patterns like GALPAT (a galloping pattern), checkerboard, and a sliding diagonal pattern are known to be excellent for testing regular structure such as random access memory and read only 


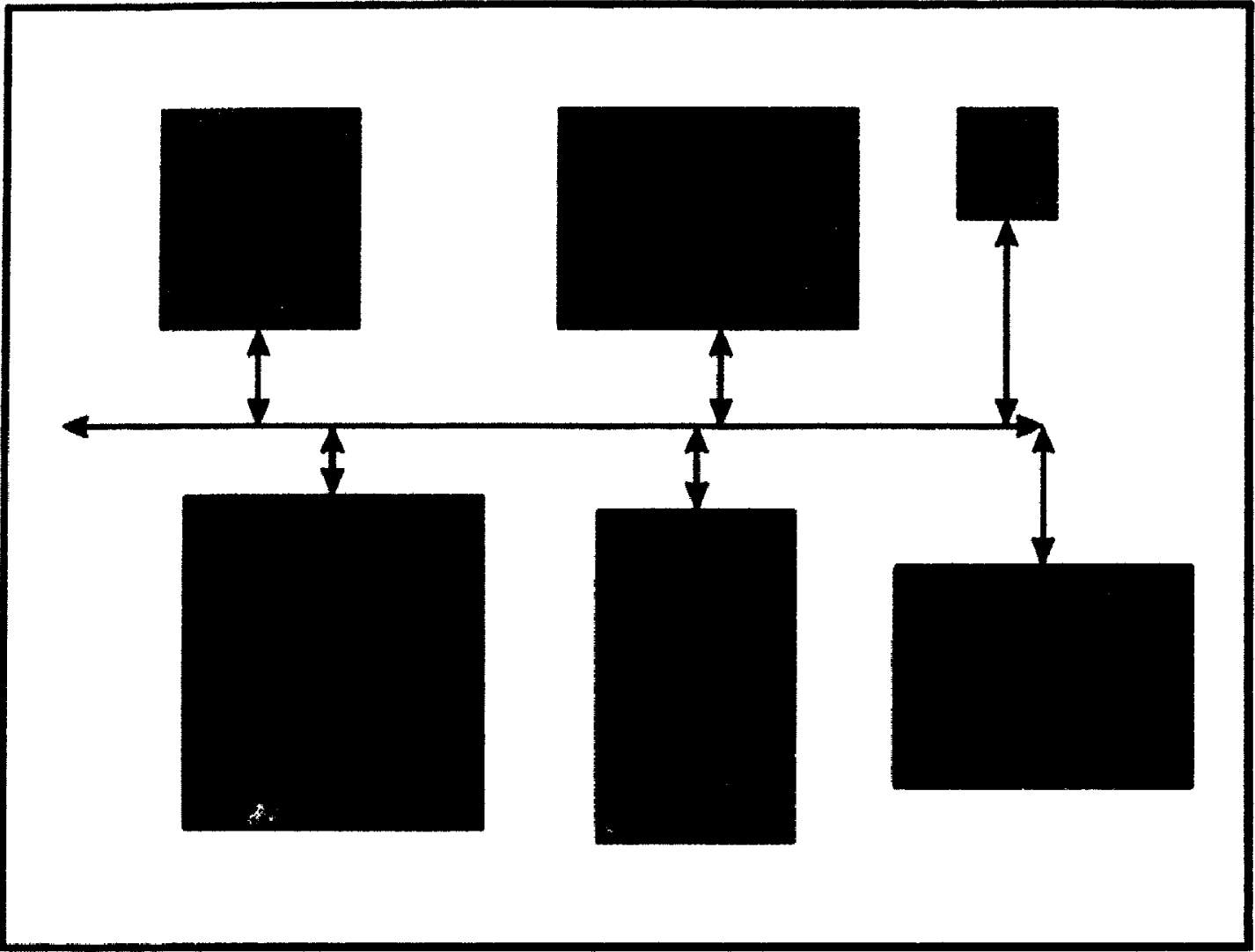

Figure 6-2: $\quad$ Test Bus Structure

memory [25]. Pseudorandom patterns are valuable for testing combinatorial logic structure [26]. Signature analysis is a way of compressing results into a signature and determining the go/no-go by comparing the signature to a predicted one.

The advantages of BIST can be summarized as follows:

1) it can simplify chip testing on- and off-board

2) testing can be conducted at-speed without external stimuli

3) it is the only way to provide at-speed testing when ASIC has a higher operating speed than the external tester that is used to test them

4) a continuous self-test can provide a reference chip operation for power ard current measurement under various supply voltages and operating temperatures 
5) it is cost eflective. typically taking $0-10^{\circ}$ o of the silicon real estate

\subsection{Radix-2 Complex Butterfly ASIC Testing Sirategy}

To choose a testing strategy for the complex butterly ASIC, its circuit stricture must first be examined. The bulk of the complex butterly ASIC is the computation pipeline which corisists of combinatorial logics and data registers. Data entering into this cascaded pipeline flows directly through to the output This type of data path architecture makes internal nodes inside the pipeline inherently visible and results in goud controllability and observability. Therefore. BIST is selected as the testing strategy for the butterfly ASIC over the gate intensive scan path testing strategy

BIST operation is enabled by two signals: the chip-select and the self-test enable. Once the self-test is activated, all the circuits go through a reset sequence. Then ranuiom vectors are generated for the a, b, c, d, and the complex twiddle factors. All are fed into the complex butterfly computation pipeline as shown in Figure 0-3. The outputs from the butterfly pipeline are compressed by a multiple input signature analyzer. The compacted signature is then compared to the predicted signature, and if there is an agreencent with the comparison, a go/no-go signal will pulse low for five clock cycles. At this point, if the self-test condition is still asserted, all circuits will return to the reset state and restart another self-test cycle. Therefore, a fixed interval pulse train of go/no-go signal will be generated in a continuous self-test. 


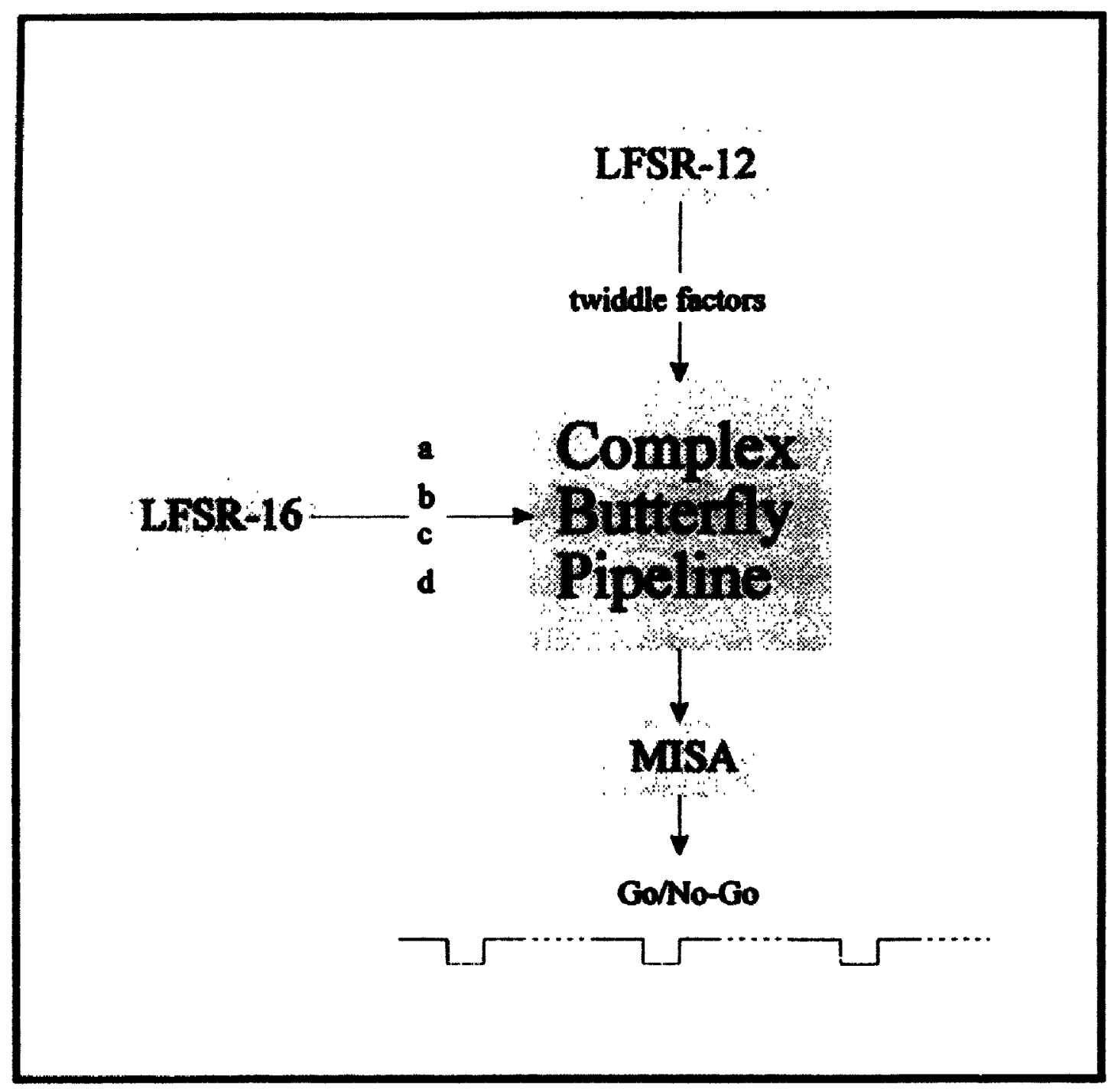

Figure 6-3: BIST for Complex Butterfly ASIC

\subsection{Preudorandom Test Vector Gemerator}

BIST includes two linear feedback shift registers for pseudorandom vector generation. One 16-bit generator supplies pseudorandom vectors for $a, b, c$, and $d$; the other 12-bit generator supplies pseudorandom vectors for the complex twiddle factors.

LFSR is a chain of registers clocked by a common clock with feedbacks from some intermediate point to the input [26]. Certain LFSR feedback arrangements can generate a maximum length pseudorandom sequence: for the 16-bit LFSR the pseudorandom 
sequence repeats every $\left(2^{16}-1\right)$ bits and for the 12-bit LFSK the pscudorandom sequence repeats every $\left(2^{12}-1\right)$ bits. Figure $6-4$ shows the maximum length I.FSR circuit implementation for the 16-bit LFSR and the 12-bit LFSR.
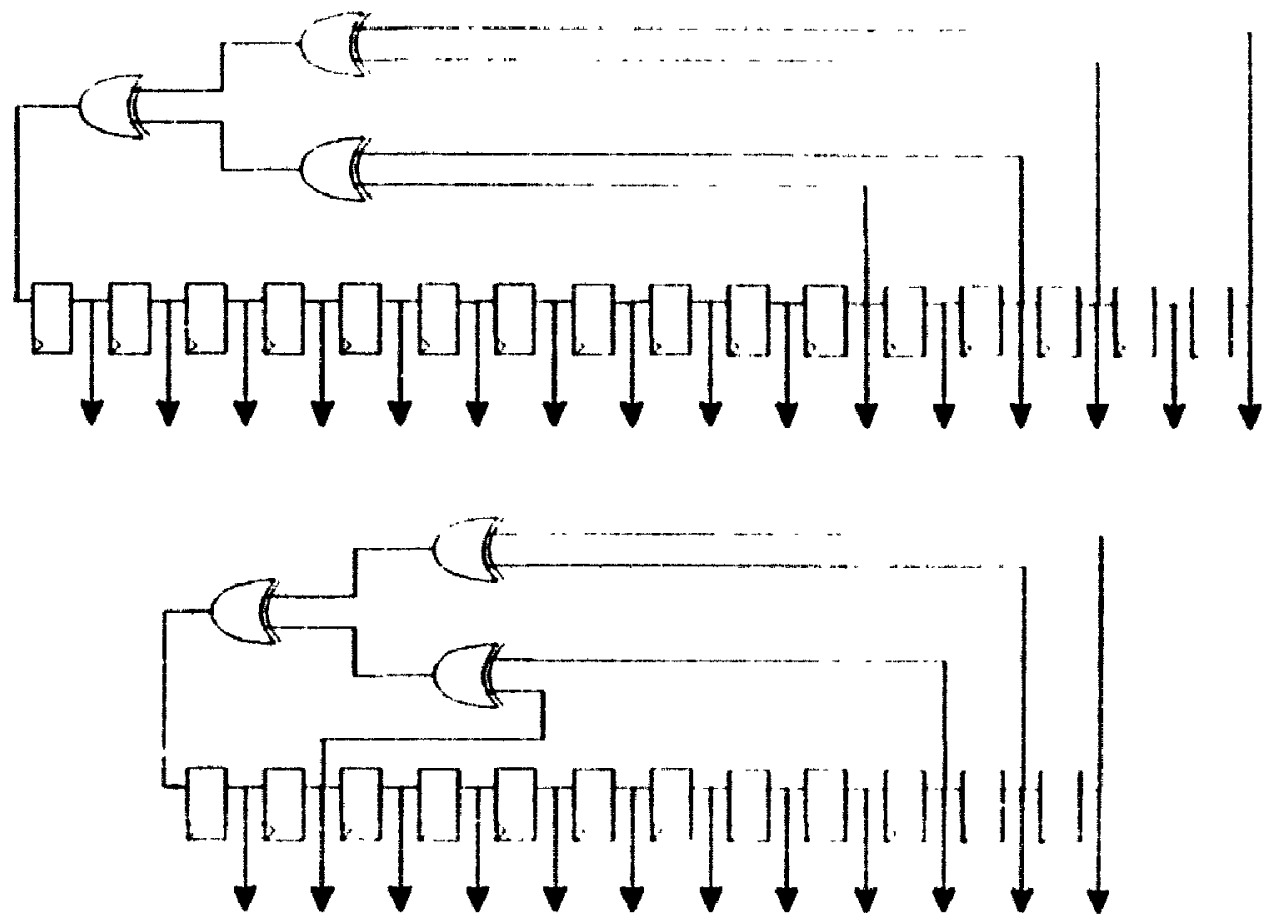

Figure 6-4: 16-bit LFSR and 12-bit LFSR Circuit Implementation

\subsection{Multiple Input Signature Analyzer}

MISA is similar to the LFSR generators but has the facility to accept external inputs. Figure 6-5 shows a portion of the MISA input facility without showing the feedbacks. Instead of passing the output directly from one register to the input of next register as in the LFSR, the output is passed through an exclusive-or gate with an external input before it is moved to the next register. 


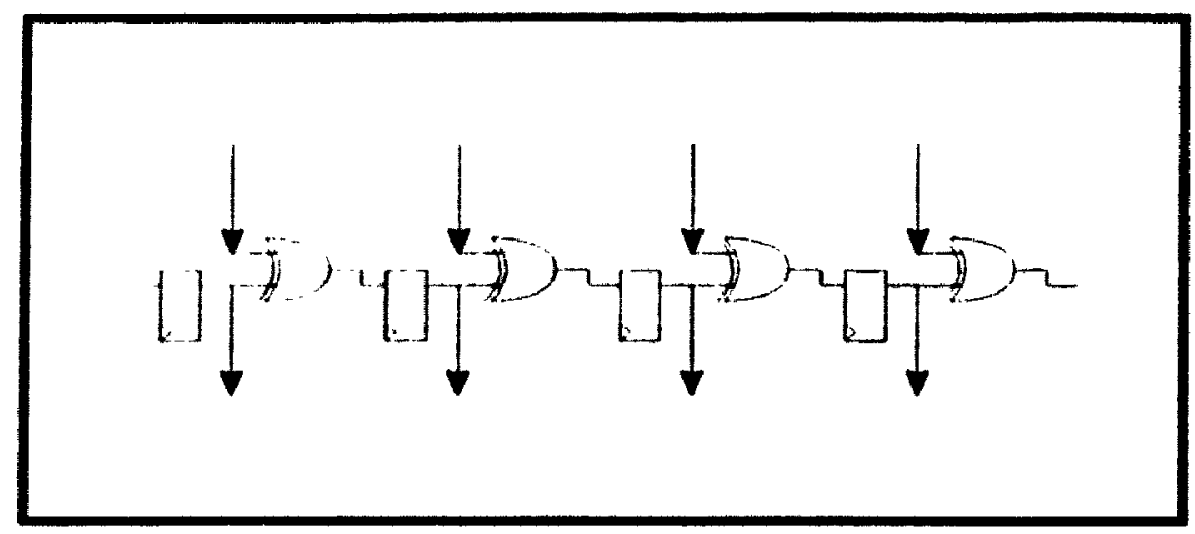

\section{Eigure 6-5: Portion of MISA - Input Facility}

There is one problem with signature analysis called signature aliasing or signature masking. Aliasing occurs when a fault signature is the same as the no-fault signature. The compacted signature is assumed to be randomly distributed; that means that the probability of signature masking is $\left[1 /\left(2^{16}-1\right)\right]$ or $.0015 \%$ for a 16-bit signature [27].

\subsection{BIST Gate Count}

The BIST gate count for the complex butterfly is 450-gate which represents approximately $3 \%$ of the final ASIC gate counts.

\subsection{Summary}

This chapter has described some "design for testability" solutions that will solved the problems in ASIC testing. Various testing strategies were discussed and the testing strategy decision for the butterfly ASIC and its BIST circuit implementations were described. 


\section{Chapter 7}

\section{CMOS Gate-Array Technology}

\subsection{Introduction}

This chapter describes the computer-aided design tools and the design process for the complex butterfly ASIC development. The design process can be divided into two parts: the fromt-end process which is performed in-house, and the back-end process which takes place at the ASIC vendor. The success of the ASIC greatly relies on the quality of the back-end layout process.

\subsection{ASIC Computer-Aided Design Tools}

The computer-aided design tools available for the complex butterlly ASIC include a Mentor Graphics schematic editor called Neted and a Mentor Graphics simulator called Quicksim that runs on an Apollo platform. Mentor Graphics is a third party tool-vendor, who supports various ASIC vendors' macro-cell libraries that include LSI Logic's LMA 9000 macro-cells library. This macro-cell library contains basic logic gates, counters, and multiplexers for use, in the schematic entry.

Once the complex butterfly architecture is defined, schematics are captured hierarchically in a bottom-up fashion by using Neted. Small functional modules are built first: for example, the bit-slice RBA adder cell, the bit-slice partial product generator, pseudorandom vector generator, and mu'tiple inpti signature analyzer. Then more complex functional modules are built by using the defined functional modules, such as the 16-bit RBA adder and 17-bit partial product generator. These high function modules ase then integrated into a higher function module and the process continues. Figure 7-1 
provides an example of a histarchical schematic capture that shows the integration of a 4-bit RBA adder.

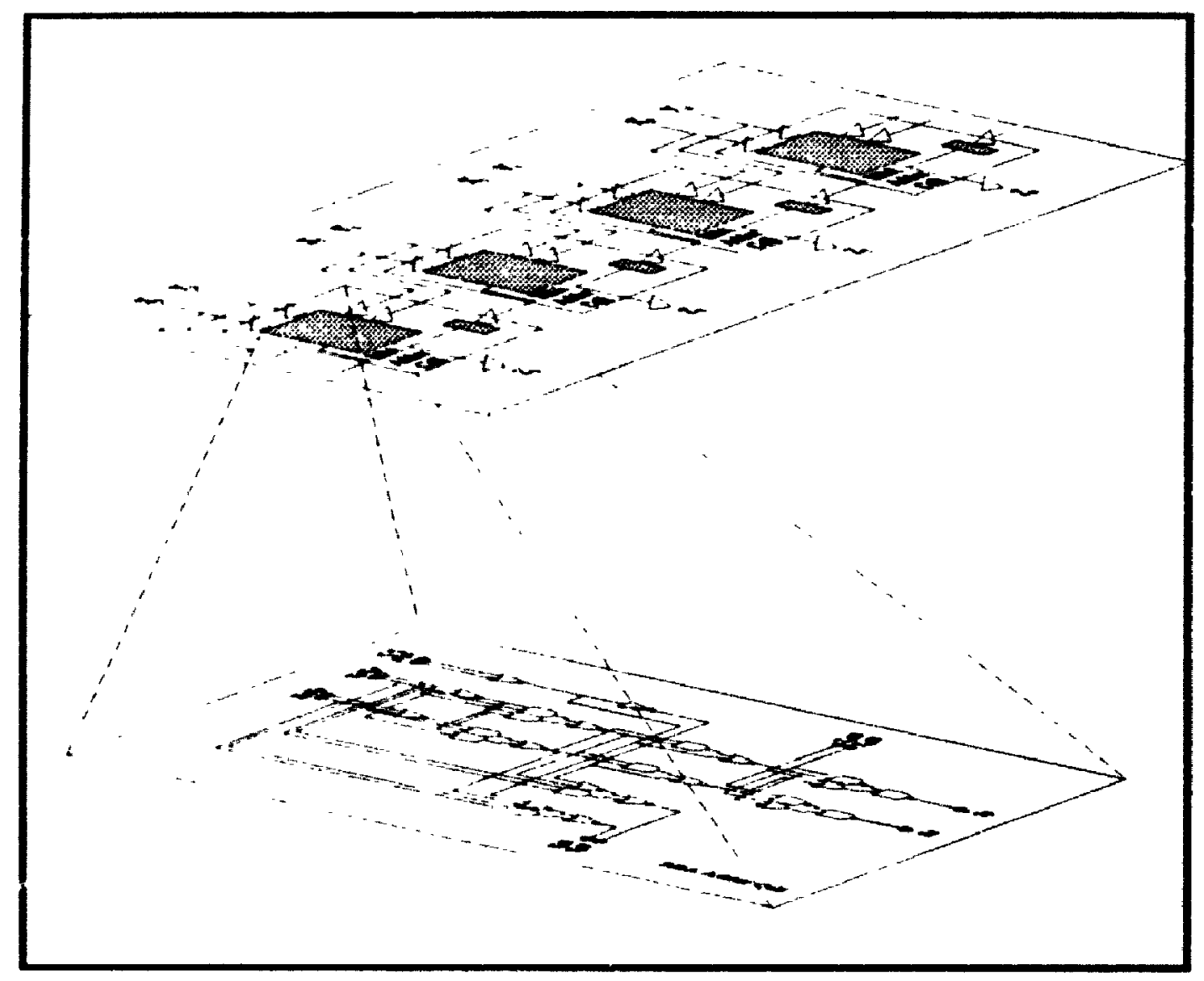

Figure 7-1: Bottom-Up Hierarchical Schematic Example.

After schematic capture, an expand process takes place which levels the hierarchical schematics into a flat netlist. A fiet netlist is equivalent to a single sheet schematic comstructed with only the basic cells: inveners, 2-input NAND gates, and 2-input OR gates. A flat netlist resembles a sea of gates.

Following the expand process, the flat netlist is compiled and each gate is appended with a fixed propagation gate delay which is based on a single capacitive load. This single capacitive load ignores the layout tracking capacitance (a function of track length) and the actual fanout loading (number of inputs driven by the output) for each 
gate. A better gate propagation delay can be estimated by using I.SI's proprictary floorplanning tool and propagation delay estimator Floorplanning allows the designer to define the placement of high level functional modules In genceral, it is suflicient to floorplan the top three levels. The propagation delay estimator takes into account this floorplanning information and the gate output fanout when gencrating the gatse propagation delays.

Quicksim is used to simulate and verify the logic function of the circuil functional simulation is performed in a bottom-up manner that uses unity propagation gate delay. First, the lower modules are simulated and verified, then higher functional modules are simulated and verified; the process continues on to the top level module. Figure 7-2 shows an example of a logic simulation for a 4-bit RBA adder.

The process of schematic entry and functional simulation is called the front-end design process. Prior to transferring the design for the back-end process, a set of test vectors must be developed. For the butterfly ASIC, three sets of test vectors were included: a self-test, a set of externally applied pseusarandom vectors identical to the selftest vectors, and a set of functional test vectors that exercise all the miscellaneous complex butterfly functions.

\subsection{Gate-array ASIC Back-End Design Process}

The gate-array ASIC back-end process which takes place at the vendor includes

- floorplanning and layout of circuits

- timing simulation and verification with back-annotated propagation delay based on the layout

- mask generation

- transfer to prototype fabrication 


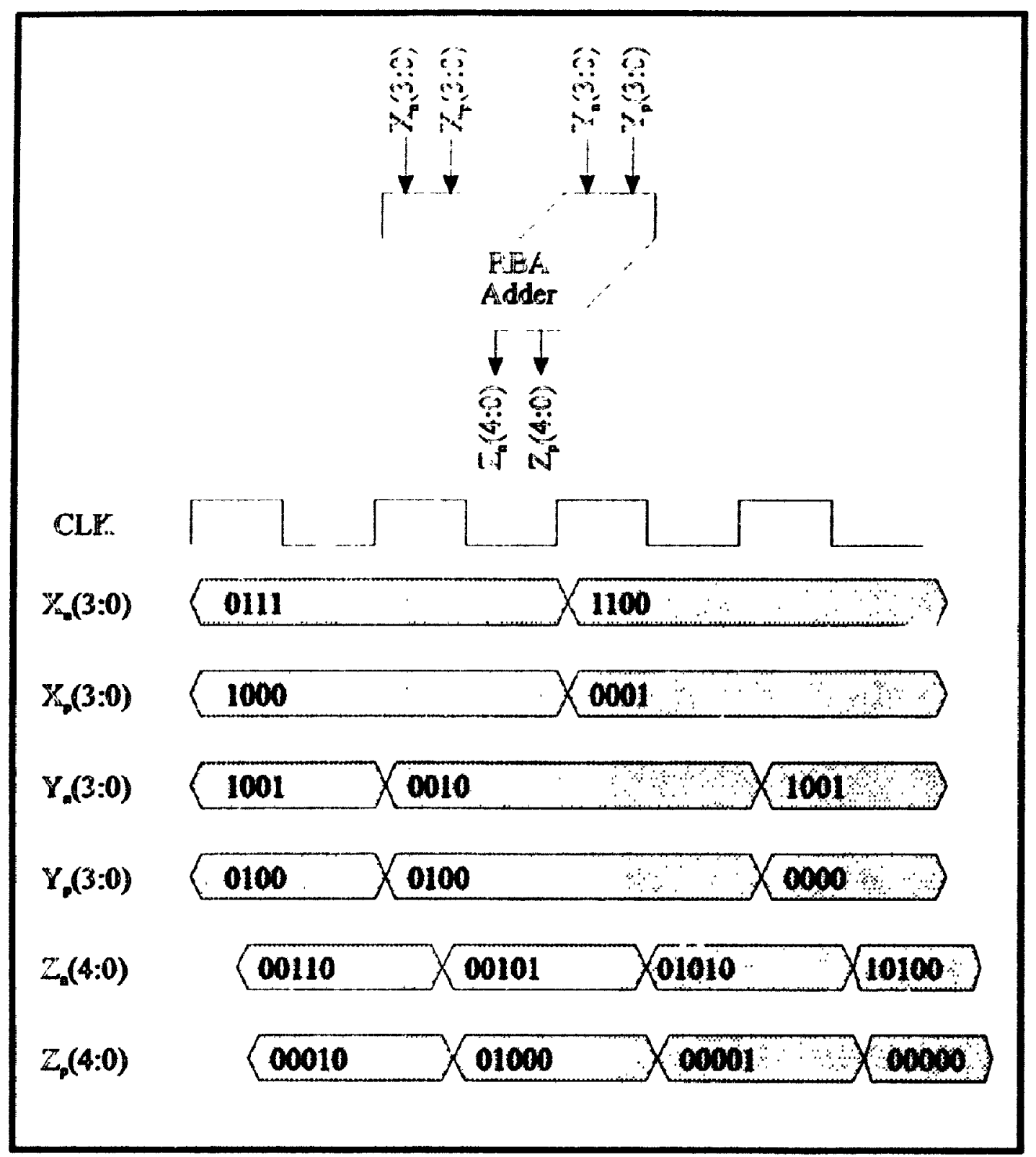

Figure 7-2: Example of Logic Functional Simulation

Floorplanning involves logical placement of circuit functional modules according to data flow. The floorplanning is generally done for the top two to three hierarchical levels. Floorplanning is meant to provide some guidance for the automatic layout tool.

Layout is performed automatically by using LSl's proprietary layout optimization program. One of the distinct features of this automatic layout program is its ability to do 
clock-tree balance distribution and clock-skew minimization for major global clocks. The purpose of skew minimization between registers is to avoid data and clock race which could lead to data setup and hold-time violation for data register. When the selup violation or hold-time violation occurs, erroneous data can be clocked in; these violations lead to incorrect operation. Figure 7-3 (a) shows the required data setup-time and hold-time with respect to the clock for a data register. Figure 7-3 (b) shows a setup time violation caused by the skewed clock.
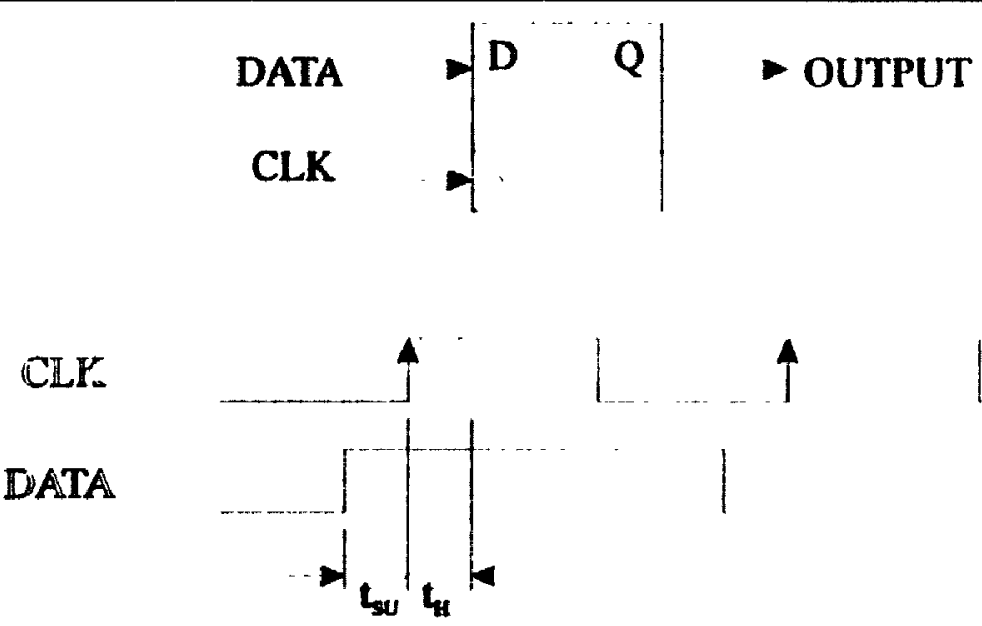

OUTPUT

DATA

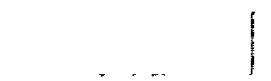

(a) Proper setup and hold time
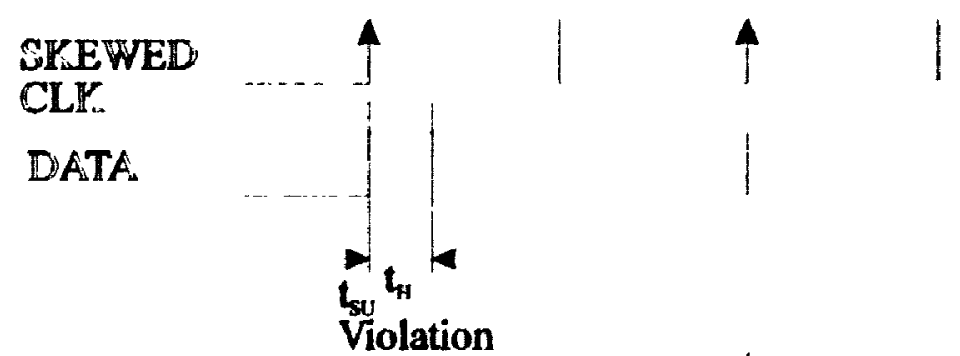

OUTPUT

Violation

(b) Setup time violation

Figure 7-3: Data Setup and Hold-Jime for Data Register 
After layout, test vectors are run with the best case, typical case, and worst case propagation delay timing. The best case, typical case, and worst case are defined by three facturs: the temperature factor, the voltage factor, and the process factor. The different cases account for variation in all three factors. Successful simulation involving the worst case condition must occur in order for the vendor to guarantee the performance of the ASIC. To verify the robustness of the layout, an extra set of test vectors based on the front-end test vectors is generated by randomly skewing the data vectors and the clock vectors by 5 nsec.

Once timing simulation is verified, two layers of metal masks are generated. Mask data and test vectors are put on a tape and then sent for prototype fabrication. After fabrication, the full set of test vectors is applied on the silicon die to identify good dies for packaging. After packaging, the full set of test vectors is applied again to verify packaged chips prior to delivery.

\subsection{Summary}

This chapter has provided a general outline of the front-end and back-end design process for the complex butterfly ASIC The in-house Mentor's Neted and Quicksim design tools used in the front-end design were discussed. The laborious and rigorous back-end process to ensure a "first-pass success" which took place in the ASIC vendor's design centre was also described. 


\section{Chapter 8}

\section{Fault Simulation Results and Prototype Test Results}

\subsection{Introduction}

Fault simulation results and prototype ASICs test results are reported in this chapter. Speed comparisons to two reported solutions are discussed. A suggestion to achieve a data sampling rate similar to that in a recent published paper by Swartzlander [14], with the present architecture is given.

\subsection{Fault Simulation Results}

BIST covers the complex butterfly computation core which represents $90 \%$ of the circuits. The remaining $10 \%$ of the circuit not covered by BIST comprise thirtcen twiddle factor registers and the a, b, c, and d input registers. One BIST test cycle requires 3,995 test vectors. A deterministic fault simulation program called Quickl'ault (a Mentor Graphics design tool) was used to determine the stuck-at fault coverage provided by the BIST, which is $87 \%$ for the entire circuit.

\subsubsection{Fault Simulation Result Discussion}

Because the $87 \%$ fault coverage result is based on the entire circuit, the fault coverage for the actual circuit under test - the complex butterfly computation core $(90 \%$ of the circuits) - equals $97 \%$.

A $97 \%$ fault coverage of the circuit under test confirms that BISI is a well-suited testing strategy for combinational type circuits and for the complex butterfly ASIC. 
Because BIST only covers $90 \%$ of the circuit, for manufacturing testing the remaining $10 \%$ of the circuit (thirteen twiddle factor registers and a, b, c, and d input registers) must be tested by additional test vectors. Two sets of test vectors, each 4,000 vectors long, are created for testing these miscellaneous circuits. Combining them with BIST creates three sets of 4,000 vectors, and maintains the number of test vectors limited by the vendor; these three sets of test vectors are projected to provide close to $97 \%$ fault coverage for the entire chip.

\subsection{Prototype Testing}

\subsubsection{Test Setup}

Prototype testing was performed on an IMS Excel-100 IC tester which supplies both test vectors identical to self-test vectors to the ASIC under test. The IC tester gains access to the ASIC via an adapter card and an interface board mounted on the tester. The average capacitive loading of the ASIC output is measured to be 116 picofarad (measured by a PM6303 LCR meter). The output capacitive loading is the sum of the IC tester probe loading ( 35 picofarad maximum), loading from the wire wrapping on the interface board, connectors on the adapter board, long traces and socket. However, the realistic capacitive loading of the ASIC output, when integrated on board where ASICs are placed an inch and a half apart and input capacitive loading is in the neighbourhood of 10 picofarad would be around 15 picofarad. Thus, the difference between the tester capacitive loading and the realistic application capacitive loading is a 100 picofarad. Because of this limitation of the tester, the measured speed ratings are adjusted for the realistic condition.

\subsubsection{Test Results}

Capacitive loading dictates the time for charging or discharging an output node which is called the output propagation delay. When pipelining data from one ASIC to 
another ASIC and both are using the same master clock. extra output propagation delay will translate to a longer master clock period by the same amount of delay. The output propagation delay adjustment for extra tester capacitive loading (reduction fiom 116 picofarad to 15 picofarad) of 9.7 nsec shrinks the measured master clock period by 9.7 nsec [28]. Tables 8-1 and 8-2 provide the measured maximum clock frequency and its corresponding clock period for twenty prototype ASICs for both a 5V and 3.3V power supply. In Table 8-1, the adjusted maximum clock frequency for each prototype is also given; it is based on a 9.7 nsec clock period correction. The average adjusted maximum frequency is $49.2 \mathrm{MHz}$ for the $5 \mathrm{~V}$ case. The average measured maximum frequency is $23.6 \mathrm{MHz}$ for the $3.3 \mathrm{~V}$ case.

Using the BIST a second test was performed. The IMS tester programs the ASIC into its self-test mode and only supplies a clock signal to the master clock pin. Then, nunning in an endless loop of self-test, with each self-test cycle 4,000 clock cycles long. the IMS tester expects a 5-clock cycle low-pulse at the go/no-go pin at the end of each self-test cycle. Figure 8-1 shows a photograph of the complex butterfly ASIC. Higures 8-2 and 8-3 show a photograph of a single go/no-go pulse, and a photograph of a go/no-go pulse train. Table 8-3 presents the maximum clock frequency and power consumption measured by the tester for a $5 \mathrm{~V}$ and a $3.3 \mathrm{~V}$ case. The measured maximum clock fiequency is $58 \mathrm{MHz}$ which implies a $29 \mathrm{Msamples} / \mathrm{sec}$ data throughput rate or a data sampling rate of $14.5 \mathrm{MHz}$.

\subsubsection{Test Result Discussion}

From the BIST test result and the non-BIST test result, the output propagation delay can be derived. From the BIST test result, the minimum clock period is 17 nsece which represents the minimum internal pipelining delay. From the non-B3ISI test Jesult, 


\begin{tabular}{|c|c|c|c|c|}
\hline $\begin{array}{c}\text { Device } \\
\#\end{array}$ & $\begin{array}{l}\text { Maximum } \\
\text { Frequency } \\
\text { (MHz) } \\
\text { at } 5 \mathrm{~V} \text { supply } \\
\text { and } 116 \mathrm{pF} \\
\text { Load }\end{array}$ & $\begin{array}{c}\text { Minimum } \\
\text { Period } \\
\text { (ns) } \\
\text { at } 5 \mathrm{~V} \text { supply } \\
\text { and } 116 \mathrm{pF} \\
\text { Load }\end{array}$ & $\begin{array}{l}\text { Adjusted } \\
\text { Maximum } \\
\text { Frequency } \\
(\mathrm{MHz}) \\
\text { for } 15 \mathrm{pF} \mathrm{Load}\end{array}$ & $\begin{array}{l}\text { Adjusted } \\
\text { Minimum } \\
\text { Period } \\
\text { (ns) } \\
\text { for } 15 \mathrm{pF} \text { Load }\end{array}$ \\
\hline 1 & 33.2 & 30.1 & 49.0 & 20.4 \\
\hline 2 & 33.1 & 30.2 & 48.8 & 20.5 \\
\hline 3 & 32.9 & 30.4 & 48.3 & 20.7 \\
\hline 4 & 33.8 & 29.6 & 50.3 & 19.9 \\
\hline 5 & 33.2 & 30.1 & 49.0 & 20.4 \\
\hline 6 & 33.1 & 30.2 & 48.8 & 20.5 \\
\hline 7 & 34.1 & 29.3 & 51.0 & 19.6 \\
\hline 8 & 32.9 & 30.4 & 48.3 & 20.7 \\
\hline 9 & 33.1 & 30.2 & 48.8 & 20.5 \\
\hline 10 & 33.7 & 29.6 & 50.3 & 19.9 \\
\hline 11 & 33.8 & 29.6 & 50.3 & 19.9 \\
\hline 12 & 32.9 & 30.4 & 48.3 & 20.7 \\
\hline 13 & 33.1 & 30.2 & 48.8 & 20.5 \\
\hline 14 & 33.2 & 30.1 & 49.0 & 20.4 \\
\hline 15 & 33.2 & 30.1 & 49.0 & 20.4 \\
\hline 16 & 33.1 & 30.2 & 48.8 & 20.5 \\
\hline 17 & 34.1 & 29.3 & 51.0 & 19.6 \\
\hline 18 & 32.9 & 30.4 & 48.3 & 20.7 \\
\hline 19 & 33.1 & 30.2 & 48.8 & 20.5 \\
\hline 20 & 33.8 & 29.6 & 50.3 & 19.9 \\
\hline Average & 33.3 & 30.0 & 49.3 & 20.3 \\
\hline
\end{tabular}

Table 8-1: Measured and Adjusted Maximum Clock Frequency for $5 \mathrm{~V}$ Case 


\begin{tabular}{|c|c|c|}
\hline $\begin{array}{c}\text { Device } \\
\#\end{array}$ & $\begin{array}{c}\text { Maximum } \\
\text { Frequency } \\
(\mathrm{MHz}) \\
\text { at } 3.3 \mathrm{~V} \text { supply } \\
\text { and } 50 \mathrm{pF} \text { Load } \\
\end{array}$ & $\begin{array}{l}\text { Minimum } \\
\text { Period } \\
\text { (ns) } \\
\text { at } 3.3 \mathrm{~V} \text { supply } \\
\text { and } 50 \mathrm{pF} \text { Load }\end{array}$ \\
\hline 1 & 24.1 & 415 \\
\hline 2 & 23.2 & 43.1 \\
\hline 3 & 23.4 & 42.7 \\
\hline 4 & 24.6 & 40.6 \\
\hline 5 & 23.3 & 43.0 \\
\hline 6 & 23.3 & 42.9 \\
\hline 7 & 23.8 & 42.1 \\
\hline 8 & 23.3 & 42.9 \\
\hline 9 & 23.2 & 43.1 \\
\hline 10 & 23.3 & 43.0 \\
\hline 11 & 24.6 & 40.6 \\
\hline 12 & 23.4 & 42.7 \\
\hline 13 & 23.2 & 43.1 \\
\hline 14 & 24.1 & 415 \\
\hline 15 & 23.3 & 43.0 \\
\hline 16 & 23.3 & 42.9 \\
\hline 17 & 23.8 & 421 \\
\hline 18 & 23.3 & 42.9 \\
\hline 19 & 23.2 & 43.1 \\
\hline 20 & 23.3 & 430 \\
\hline Average & 23.6 & 42.5 \\
\hline
\end{tabular}

Table 8-2: Measured Maximum Clock Frequency fơr 3.3V Case 
the incasured minimum clock period is 30 nsec which represents the internal pipelining delay and the output propagation delay. The 13 nsec difference between the BIST and the non-BIST period represents the output propagation delay that resulted from the tester load. The projected tester load given in the LSI data book [28] for a B2 output driver with a 13 nsec buffer delay is 100.6 picofarad. This load projection is in agreement with the measured capacitance of 116 picofarad.

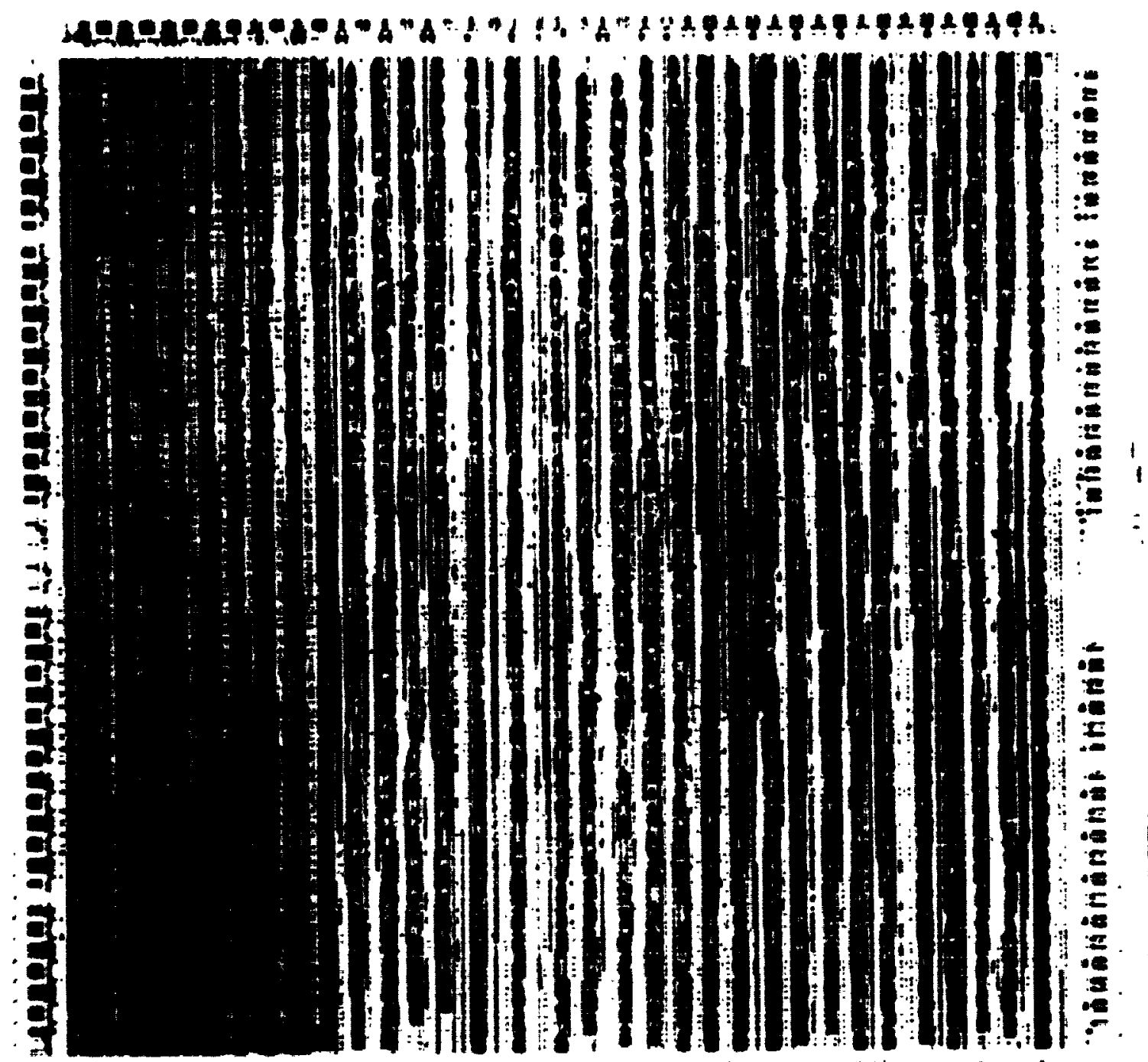

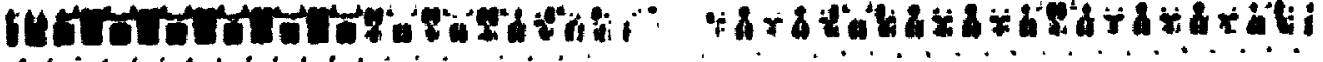

Figure 8-1: The Radix-2 Complex Butterfly ASIC 


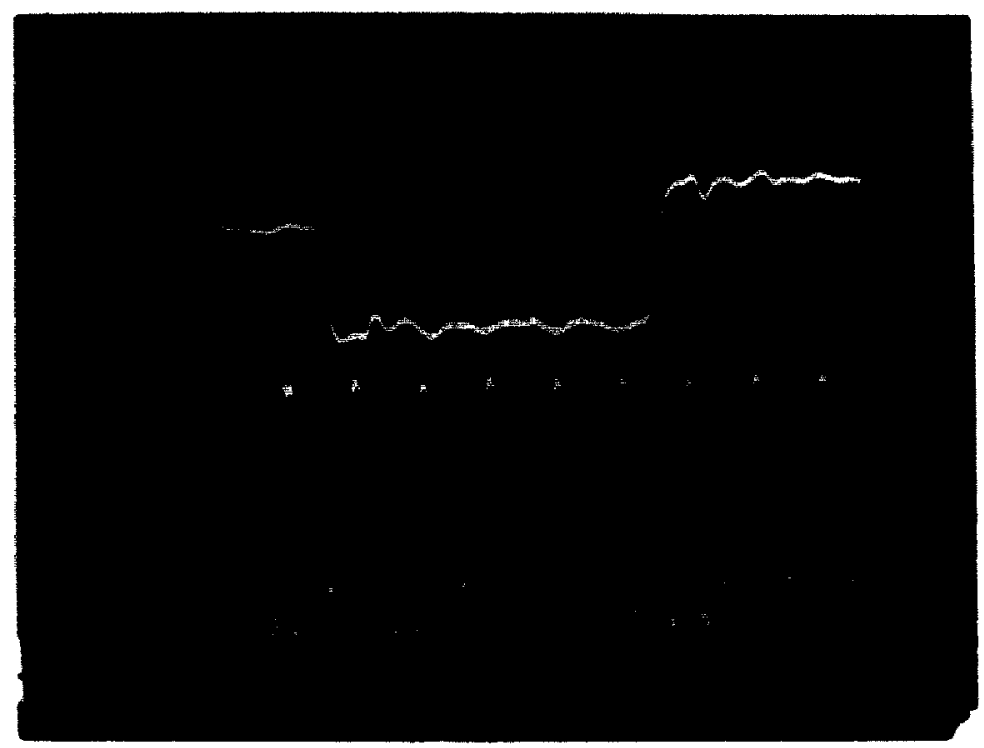

Figure 8-2 A Single Go/No-Go Pulse

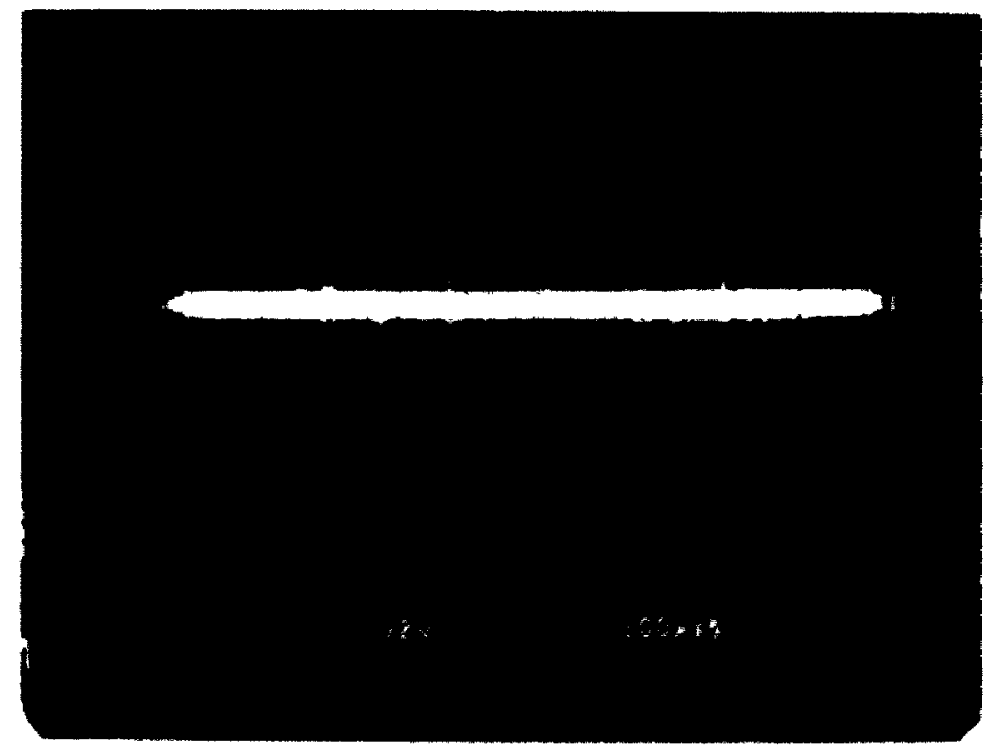

Figure 8-3 A Go/No-Go Pulse Train 


\begin{tabular}{|c|c|c|c|c|}
\hline $\begin{array}{c}\text { Supply } \\
\text { Voltage } \\
(\text { Volt) }\end{array}$ & $\begin{array}{c}\text { Clock } \\
\text { Speed } \\
(\mathrm{MHz})\end{array}$ & $\begin{array}{c}\text { Data Throughput } \\
\text { Rate (Msamples/sec) }\end{array}$ & $\begin{array}{c}\text { Data Sampling Rate } \\
(\mathrm{MHz})\end{array}$ & $\begin{array}{c}\text { Power } \\
\text { Consumption } \\
\text { (Watt) }\end{array}$ \\
\hline 3.3 & 38 & 19 & 9.5 & .76 \\
\hline 5 & 58 & 29 & 14.5 & 2.9 \\
\hline
\end{tabular}

\section{Table 8-3: BIST Test Results for 3.3V and 5V}

\subsection{Speed Comparison with Existing Reported Solutions}

Fox [13] reported a $10 \mathrm{MHz}$ data sampling rate FFT that uses two complex multiplier chips and one accumulator/reordering chip. Fox's chip-set also employs a self-test and is based on a 2-micron technology. In comparison, the ASIC developed in this thesis has a higher than Fox's data sampling rate and is a single-chip solution for the entire complex butterfly.

Plessey Semiconductors has also published an equivalent DIT radix-2 complex butterly processor solution. Similar to Fox, it also offers a three-chip solution: one PDSP16116/A 16x16 bit complex multiplier accompanied by two PDSP16318As complex adders. The data sampling rate is $20 \mathrm{MHz}$ with a 3-watt power consumption [15]. The implementation technology is unknown. Al the end of 1992, there are still no samples for these chip-sets. If the ASIC gate resource constraint is removed, the present architecture can be duplicated so that the data sampling rate will double to $24.6 \mathrm{MHz}$ which will be higher than that of Plessey's.

The complex butterlly ASIC implemented in this thesis runs with a daia sampling rate equal to one quarter of the master clock rate. This data sampling rate can be doubled 
to half the master clock rate if the pipelining of the teal and imaginary results is icmoved and if they are evaluated in parallel. With the present architecture, this data sampling tate doubling could be accomplished by duplicating the computation cors thus, the architecture proposed in this thesis allows for easy migration to doubling the data sampling rate based on the same master clock rate that uses a $30,0(0)$-gate resource

\subsection{Future Development}

This thesis proposes two suggestions for future development of a high-speed radix-2 complex butterfly ASIC. These suggestions would potentially increase the current complex butterfly ASIC data sampling rate by six times. First, increase parallelism for evaluating the real and imaginary outputs by duplicating the present computing core would make use of a 30,000-gate ASIC. This parallelism would allow the data sampling rate to double. Second, migrate to a .7-micron CMOS technology from the 1 -micron CMOS technology - a move which would allow a three-tine increase in the data sampling rate. If these two suggestions are taken, the projected data sampling rate is $75 \mathrm{MI}$ \% which is similar to the projected data sampling rate of $80 \mathrm{MHz}$ pubiisiced in a recent research by Swartzlander [14]. 


\section{Conclusion}

The DIT radix-2 complex butterfly ASIC described in this thesi: has achieved a first-pass success. By using redundant binary arithmetic, Booth's algorithm, and paralle! pipeline architecture techniques, the complex butterfly ASIC achieves a $12.3 \mathrm{MHz}$ data sampling rate. The BIST fault coverage is $\mathbf{9 7 \%}$ for the circuit under test and is $\mathbf{8 7 \%}$ for the entire ASIC.

The present computation core of the complex butterfly ASIC easily lent itself to doubling the data sample rate by duplicating the present parallel pipeline architecture. Combining this duplication and migration/promoting to a sub-micron technology, the data sampling rate can reach $75 \mathrm{MHz}$ or $150 \mathrm{Msamples} / \mathrm{sec}$.

The immediate applications of the complex butterfly ASIC are: as a single complex butterfly processor, to form a high-speed systolic FFT, and to form a single or parallel pipeline FFT with a switch ASIC. The complex butterfly ASIC provides compact and high-speed operations to these digital signal processing applications.

The present ASIC met $98 \%$ of the $12.5 \mathrm{MHz}$ target data sampling rate; future development of the complex butterfly ASIC with expanded resources could launch the rate to $75 \mathrm{MHz}$ which can definitely accommodate the on-board satellite processing application and ultinately even higher speed applications. 


\section{References}

[1] C.P.S. Yeung, "A Cascadeable Pipelined Fast fourier Transform Switch With Built-In Self-Test", Master Thesis, Carleton University, Ottawa, Ontario, 1901.

[2] G.D. Bergland, "A Guided Tour of the Fast Fourier Transtiorm", $11 \% 1 \%$. Syectrum. pp. 41-52, July 1969 .

[3] M.S. Roden, "Signal Analysis".

[4] R.B. Randall, "Frequency Analysis", ISBN 87-87355-07-8, Sept 1987.

[5] D.R. Bungard, L. Lau, and T.L. Rorabaugh, "Programmable FFT Processors for Wide-Bandwidth HF Spread-Spectrum Communications and Radar Signal Processing", 1989 International Conference on Acoustics, Speech and Signal Processing, p. 1357-9 vol.2, 1989.

[6] S. Bellini, C. Molinari, and G. Tartara, "On Board Demodulation in Multicarrier

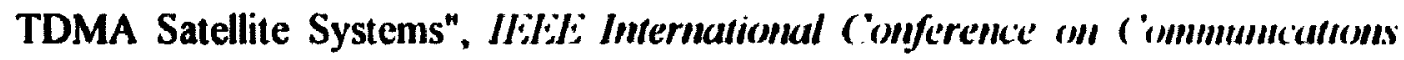
'88, pp. 499-503, vol 1, 1988.

[7] T. Alberty, G. Bjornstrom, H. Eyssele, H. Gockler, and V Ilespelt, "I)igital (OnBoard Multicarrier Demodulator for Mobile Satellite Communications", II:1:1: Imternational (onference on C'ommunicalions' '8\$, pp. 516-520, vol 1, 1988

[8] W.H. Yim, C.C.D. Kwan, F.P. Coakley, and B.G IEvans, "Multi-Carrier Demodulators for On-Board Processing Satellites", Imteriatiomal .Iourmal of Satellite Communicatıons, pp. 243-251, vol 6, Aug 1988. 
[9] P.D. Stigall, R.E. Zimer, and V.T. Pham, "A performance study of 16-bit microcomputer-implemented FFT algorithms", IELE Micro, pp. 61-65, Nov 1982.

[10] S.M. Said, and K.R. Dimond, "Improved Implementation of FFT Algorithm on a High-Performance Processor", Electronic Letter, pp. 347-349, vol 20, no 8, 1984.

[11] N.K. Riedel, D.A. Mcaninch, C. Fisher, and N.B. Goldstein, "A Signal Processing Implementation for an IBM-PC-Based Workstation", IEEE Micro, pp. 52-67, 1985

[12] K.L. Kloker, B. Lindsley, N. Baron, and G. Sohie, "Efficient FFT Implementation on an IEEE Floating-Point Digital Signal Processor", 1989 International Conference on Acoustics, Speech and Signal Processing, p. 1302-5 vol.2, 1989.

[13] J. Fox, G. Surace, and P.A. Thomas, "A Self-Testing 2- $\mu \mathrm{m}$ CMOS Chip Set for JFT Applications", IEEE Journal of Solid-State Circuits, pp. 15-19, vol SC-22, no I, Feb 1987

[14] Swartzlander, E.E., Jr; Jain, V.K.; Hikawa, H., "A Radix-8 Wafer Scale FFT Processor". Journal of VLSI Signal Processing, pp. 165-76, vol 4, May 1992.

[15] "Digital Signal Processing IC Handbook", GEC Plessey Semiconductors, November 1990.

[16] Pipeline FFT study, ( RC internal documem.

[17] A. Oppenheim and R. Schafer, "Digital Signal Processing", Prentice-Hall.

[18] N Takagi, H. Yasuura, S. Yajima, "High-Speed VLSI Multiplication Algorithm with a Redundant Binary Addition Tree", IEEE Trans. on (omputers, pp.789-796, vol $\left({ }^{\circ}-34\right.$, no 9, Sept. 1985. 
[19] Y. Harata, Y. Nakamura, H. Nagase, M. Takigawa and N. Takagi, "I ligh Speed Multiplier Using a Redundant Binary Addition Tree". II:LE: Imermatiomal Conference on ('omputer Arithmetic, pp 105-170, 1984.

[20] S. Kuninobu, T. Nishiyama, H. Edamatsu, T. Taniguchi, and N. Takagi, "Design of High Speed MOS Multiplier and Divider Using Redundant Binary Representation", Proc. of the 8ih Symposium on ('omputer Arithme'tc: pp 80-80. May 1987.

[21] A. Avizienis, "Signed-Digit Number Representation for Fast Parallel Arithmetic". IRE Trans. on Electronic Computers, pp 389-400, 1961.

[22] H.T. Nagle, S.C. Roy, C.F. Hawkins, M.G. Mcnamer, and R.R. Iritzemeier, "Design for Testability and Built-In Self Test: A Review". Il:li: Iruns. col Industrial Electronics, pp. 129-140, vol. 36, no. 2, May 1989.

[23] C. Hawkins et. al., "The VLSI Circuit Test Problem - A Tutorial", Ilili: Trums. om Indusirial Electromics, pp. 111-138, vol.36, no.2, May 1989

[24] "Chip-Level Full-Scan Design Methodology Guide", Ls: logic, Mar 1989

[25] D. Aadsen, H. Scholz, and Y. Zorian, "Automated BIST for Regular Structures Embedded in ASIC Devices", AT\&T Technical Journal, pp. 97-109, May 19\%)

[26] P.H. Bardell, W.H. McAnney, and J Savir, "Built-l"n Test forr VI.SY: Pseudorandom Techniques", John Wiley \& Sons, 1987.

[27] D.M. Miller, and S. Zhang, "Aliasing in Multiple-Input Data Compactors", I'rox: Cancatian Conf. on Elecirical \& ('omputer ling., pp. 347-351, Sept 1989.

[28] "Databook for LMA9000 Micro Array Technology", LSS/ I Aggkc, Sept 1987 
[29] P D Hortensius, R.D. Mcleod, W. Pries, D.M. Miller, and H.C. Card, "Cellular Automata-Based Pseudorandom Number Generators for Built-in Self-Test", IEEE Trums. ('ompuler-Arded/lesign, pp. 842-859, vol. 8, no. 8, Aug 1989.

[30] D.H. Miller, "Keview of Built-In Self-Test Methodologies", Proc. Canadian Conf. om lilectrical de ('omputer ling., pp. 375-378, Nov 1988.

[31] Panel Discussion, "The Challenges of Self-test", IEEE Design \& Test of ('omputers, pp. 46-54, Feb 1990.

[32] J.M. Glass, "An Efficient Method for Improving Reliability of a Pipeline FFT". II:Y:E Trans. on ('omputers, pp. 1017-1020, vol C29, no 11, Nov 1980.

[33] G.K. Ma, and F.J Tay'or, "Multiplier Policies for Digital Signal Processing", IEEE ASSP' Magazime, vol 7, no. 1, pp. 6-20, Jan 1990. 

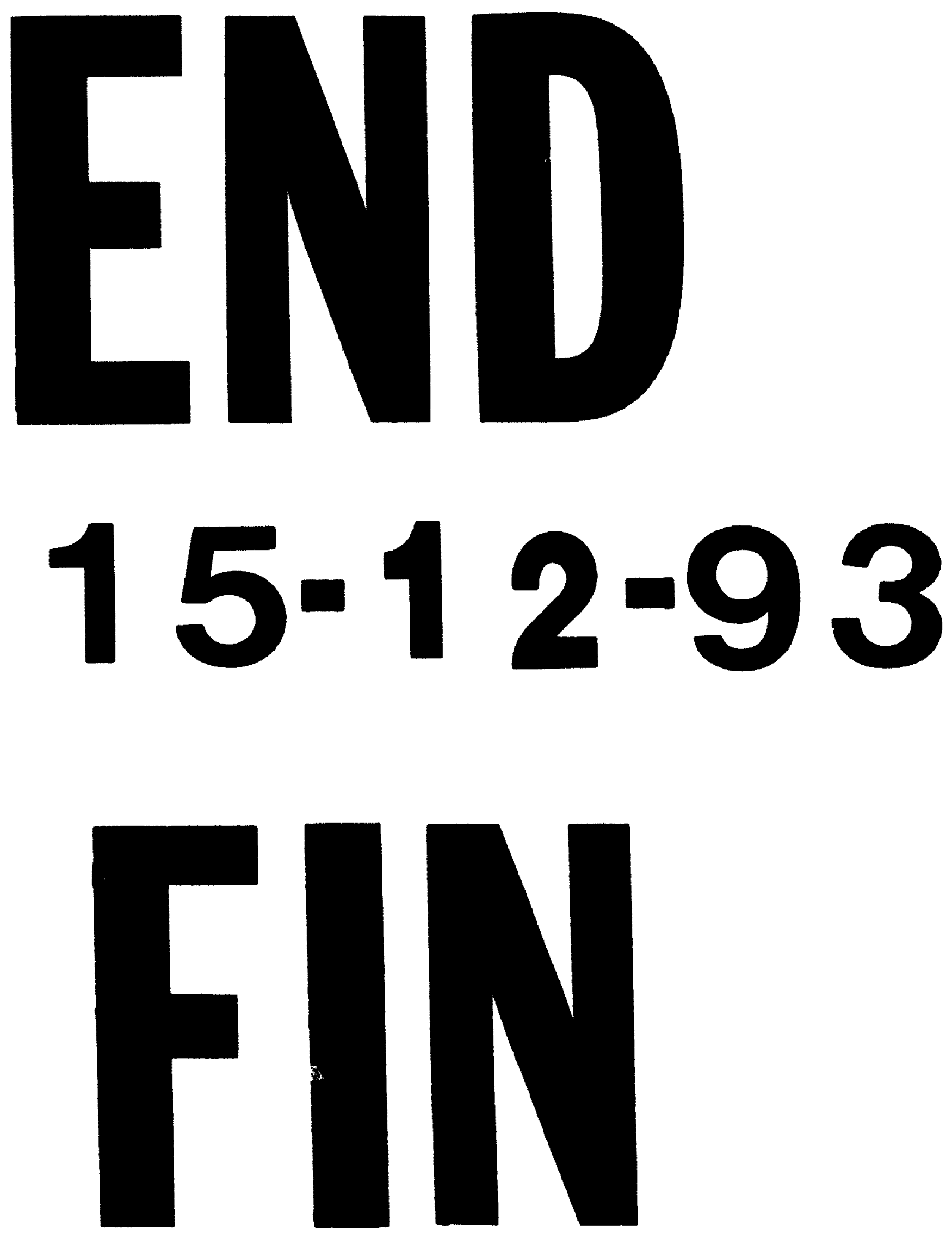